\title{
A NON-COMMUTATIVE FEJÉR THEOREM FOR CROSSED PRODUCTS, THE APPROXIMATION PROPERTY, AND APPLICATIONS
}

\author{
JASON CRANN ${ }^{1}$ AND MATTHIAS NEUFANG ${ }^{1,2}$
}

\begin{abstract}
We prove that a locally compact group has the approximation property (AP), introduced by Haagerup-Kraus [21], if and only if a non-commutative Fejér theorem holds for its associated $C^{*}$ - or von Neumann crossed products. As applications, we answer three open problems in the literature. Specifically, we show that any locally compact group with the AP is exact. This generalizes a result by Haagerup-Kraus [21], and answers a problem raised by Li in [27]. We also answer a question of Bédos-Conti [4] on the Fejér property of discrete $C^{*}$-dynamical systems, as well as a question by Anoussis-Katavolos-Todorov [3] for all locally compact groups with the AP. In our approach, we develop a notion of Fubini crossed product for locally compact groups and a dynamical version of the slice map property.
\end{abstract}

\section{INTRODUCTION}

In his seminal work on Fourier series in the early $20^{\text {th }}$ century, Fejér established, under appropriate conditions, the approximation of a function by the Cesàro sum of its Fourier series [16]. More specifically, if $f \in L^{\infty}(\mathbb{T})$ with Fourier series $S_{N}(f)(t)=\sum_{n=-N}^{N} \hat{f}(n) e^{i n t}$, then

$$
\frac{1}{N} \sum_{n=0}^{N-1} S_{n}(f)=F_{N} * f \rightarrow f
$$

weak* (uniformly if $f \in C(\mathbb{T})$ ), where $F_{N}(t)=\frac{1}{N} \sum_{n=0}^{N-1} \sum_{k=-n}^{n} e^{i k t}$ is Fejér's kernel. Shortly after he gave explicit examples of continuous periodic functions whose Fourier series do not converge pointwise [17].

We may interpret the Cesàro convergence (1) through pointwise multiplication under the Fourier transform: the sequence $\left(\hat{F}_{N}\right)$ forms a bounded approximate identity for the Fourier algebra $A(\mathbb{Z})$, and we have $\hat{F}_{N} \cdot x \rightarrow x$ weak $^{*}$ for any $x \in V N(\mathbb{Z}) \cong L^{\infty}(\mathbb{T})$ (uniformly if $x \in C_{\lambda}^{*}(\mathbb{Z}) \cong C(\mathbb{T})$ ), where $\cdot$ is the canonical pointwise action of $A(\mathbb{Z})$ on $V N(\mathbb{Z})$. It follows that

$$
x=w^{*}-\lim _{N} \frac{1}{N} \sum_{n=0}^{N-1} \chi_{[-n, n]} \cdot x=w^{*}-\lim _{N} \frac{1}{N} \sum_{n=0}^{N-1} \sum_{k=-n}^{n} \tau\left(x \lambda(k)^{*}\right) \lambda(k)
$$

provides a Fejér representation for any $x$ in the von Neumann crossed product $V N(\mathbb{Z})=\mathbb{Z} \bar{\rtimes}$ through an explicit linear combination of translation operators, where $\tau$ is the canonical tracial state on $V N(\mathbb{Z})$. Note the appearance of a $\mathrm{F} \varnothing$ lner sequence for $\mathbb{Z}$, linking the Cesàro summability to amenability of $\mathbb{Z}$.

Similar Fejér type representations exist for non-trivial crossed products $G \bar{\rtimes} M$ where $G$ is a locally compact abelian group acting on a von Neumann algebra $M$ (see, for instance, [26, 42] or $[34, \S 7.10])$, where the coefficients are now $M$-valued. In this setting, the Cesàro sum is replaced by a suitable average over a bounded approximate identity in $L^{1}(\widehat{G}) \cong A(G)$, again linking Fejér representations to amenability of $G$.

2010 Mathematics Subject Classification. 47L65, 46L55, 46L07.

Key words and phrases. Crossed products; locally compact groups; the approximation property; exactness. 
If $G$ is a discrete group acting on a von Neumann algebra $M$, then the "Fourier series"

$$
\sum_{s \in G} E\left(x u(s)^{*}\right) u(s)
$$

is in general not strongly or even weak* convergent to $x$ for every $x \in G \bar{\rtimes} M$, even in the case of $\mathbb{Z}$ acting trivially on $\mathbb{C}$, as mentioned above. Here, $E: G \bar{\rtimes} M \rightarrow M$ is the canonical conditional expectation, and $u(s)$ is the image of the regular representation in the crossed product. Summability properties of (2) and related questions concerning the Fourier analysis of $C^{*}$ - and von Neumann crossed products have been studied in detail by many authors (see, for instance, $[4,5,11,14$, 18, 28, 29, 30, 31, 38, 41, 42]). In particular, Fejér type representations for elements of crossed products have been considered over (weakly) amenable discrete groups [4, 5, 14, 18, 42]. In this paper, we complete one aspect of this line of work by showing that Fejér representability in crossed products is equivalent to the approximation property (AP) of the underlying locally compact group, as introduced by Haagerup-Kraus [21]. More precisely, we establish an explicit non-commutative Fejér representation for elements of $C^{*}$ - and von Neumann crossed products over arbitrary locally compact groups $G$ with the AP. Conversely, if every element of every ( $C^{*}$ - or von Neumann) crossed product admits such a Fejér representation, then $G$ necessarily has the AP. (See Theorems 4.1 and 4.10.)

As applications of our Fejér representation, we

(1) generalize a result by Haagerup-Kraus [21] for discrete groups to all locally compact groups; indeed we prove that every locally compact group with the AP is exact, thus answering Problem 9.4 (1) raised in K. Li's PhD thesis [27];

(2) answer a question of Bédos-Conti [4] on the Fejér property of $C^{*}$-dynamical systems over discrete groups with the AP, thus generalizing the corresponding result of [4] for weakly amenable groups.

(3) answer a question raised by Anoussis-Katavolos-Todorov [3], for all locally compact groups with the AP, on the structure of $V N(G)$-bimodules in $\mathcal{B}\left(L^{2}(G)\right)$, generalizing the corresponding result of [3] for abelian, compact, and weakly amenable discrete groups.

The main contribution of our article, i.e., the explicit representation of elements in crossed products, will, in our view, lead to a number of additional applications, examples of which are given near the end of the paper.

Structurally, we begin in section 2 with preliminaries on operator space tensor products and dynamical systems. In section 3 we develop a notion of Fubini crossed product, and we introduce an associated dynamical notion of the slice map property for actions of locally compact groups on $C^{*}$ - and von Neumann algebras. Section 4 is devoted to the proof of our non-commutative Fejér respresentation, and section 5 contains the aforementioned applications.

\section{Preliminaries}

2.1. Operator space tensor products. Throughout the paper we let $\widehat{\otimes}, \otimes^{\vee}$ and $\otimes^{h}$ denote the operator space projective, injective, and Haagerup tensor products, respectively. Recall that for $C^{*}$-algebras $A$ and $B$, the injective tensor product $A \otimes^{\vee} B$ coincides with the spatial $/ \mathrm{minimal}$ tensor product $A \otimes_{\min } B$ (see, e.g., [12, Proposition 8.1.6]). The algebraic and Hilbert space tensor products will be denoted by $\otimes$, the relevant product being clear from context. The weak* spatial tensor product will be denoted by $\bar{\otimes}$. On a Hilbert space $H$, we let $\mathcal{K}(H), \mathcal{T}(H)$ and $\mathcal{B}(H)$ denote the spaces of compact, trace class, and bounded operators, respectively.

For dual operator spaces $X^{*} \subseteq \mathcal{B}(H)$ and $Y^{*} \subseteq \mathcal{B}(K)$, the weak*-Haagerup tensor product $X^{*} \otimes{ }^{w^{*} h} Y^{*}$ is the space of $u \in \mathcal{B}(H) \bar{\otimes} \mathcal{B}(K)$ for which there exist an index set $I$ and $\left(x_{i}\right)_{i \in I} \subseteq X$ and $\left(y_{i}\right)_{i \in I} \subseteq Y$ satisfying $\left\|\sum_{i} x_{i} x_{i}^{*}\right\|,\left\|\sum_{i} y_{i}^{*} y_{i}\right\|<\infty$ and $u=\sum_{i} x_{i} \otimes y_{i}$, where each sum is 
understood in the respective weak* topologies. Then

$$
\|u\|_{w^{*} h}:=\inf \left\{\left\|\sum_{i} x_{i} x_{i}^{*}\right\|,\left\|\sum_{i} y_{i}^{*} y_{i}\right\| \mid u=\sum_{i} x_{i} \otimes y_{i}\right\}
$$

and the infimum is actually attained [6, Theorem 3.1]. There are corresponding matricial norms on $M_{n}\left(X^{*} \otimes^{w^{*} h} Y^{*}\right)$ giving an operator space structure on $X^{*} \otimes^{w^{*} h} Y^{*}$ which is independent of the weak* homeomorphic inclusions $X^{*} \subseteq \mathcal{B}(H)$ and $Y^{*} \subseteq \mathcal{B}(K)$. Moreover, $\left(X \otimes \otimes^{h} Y\right)^{*} \cong X^{*} \otimes^{w^{*} h} Y^{*}$ completely isometrically [6, Theorem 3.2]. In particular, by [12, Proposition 9.3.4], for any Hilbert space $H$

$$
\mathcal{B}(H) \cong(\mathcal{T}(H))^{*} \cong\left(\left(H_{c}\right)^{*} \otimes^{h} H_{c}\right)^{*} \cong H_{c} \otimes^{w^{*} h}\left(H_{c}\right)^{*}
$$

completely isometrically, where $H_{c}$ refers to the column operator space structure on $H$. Note that the $w^{*}$-Haagerup tensor product of dual operator spaces coincides with the extended Haagerup tensor product (see [13]).

Given dual operator spaces $X^{*} \subseteq \mathcal{B}(H)$ and $Y^{*} \subseteq \mathcal{B}(K)$, the normal Fubini tensor product $X^{*} \bar{\otimes}_{\mathcal{F}} Y^{*}$ is given by

$$
X^{*} \bar{\otimes}_{\mathcal{F}} Y^{*}=\left\{T \in \mathcal{B}(H \otimes K) \mid(\omega \otimes \mathrm{id})(T) \in Y^{*},(\mathrm{id} \otimes \rho) \in X^{*}, \omega \in \mathcal{T}(H), \rho \in \mathcal{T}(K)\right\} .
$$

Clearly, $X^{*} \bar{\otimes} Y^{*} \subseteq X^{*} \bar{\otimes}_{\mathcal{F}} Y^{*}$. A dual operator space $X^{*}$ is said to have the dual slice map property if $X^{*} \bar{\otimes} Y^{*}=X^{*} \bar{\otimes}_{\mathcal{F}} Y^{*}$ for all operator spaces $Y$. It is known that $X^{*}$ has the dual slice map property if and only if $X$ has the operator space approximation property (see [12, Theorem 11.2.5]).

2.2. Dynamical systems. Let $G$ be a locally compact group. The adjoint of convolution * : $L^{1}(G) \widehat{\otimes} L^{1}(G) \rightarrow L^{1}(G)$ is a co-associative co-multiplication $\Gamma: L^{\infty}(G) \rightarrow L^{\infty}(G) \bar{\otimes} L^{\infty}(G)$ satisfying $\Gamma(f)(s, t)=f(s t)$, for all $f \in L^{\infty}(G)$. There are left and right fundamental unitaries $W, V \in \mathcal{B}\left(L^{2}(G \times G)\right)$ which implement $\Gamma$ in the sense that

$$
\Gamma(f)=W^{*}\left(1 \otimes M_{f}\right) W=V\left(M_{f} \otimes 1\right) V^{*}, \quad f \in L^{\infty}(G) .
$$

They are given respectively by

$$
W \xi(s, t)=\xi\left(s, s^{-1} t\right), \quad V \xi(s, t)=\xi(s t, t) \Delta(t)^{1 / 2}, \quad s, t \in G, \xi \in L^{2}(G \times G),
$$

where $\Delta$ is the modular function of $G$. These fundamental unitaries are intimately related to the left and right regular representations $\lambda, \rho: G \rightarrow \mathcal{B}\left(L^{2}(G)\right)$ given by

$$
\lambda(s) \xi(t)=\xi\left(s^{-1} t\right), \quad \rho(s) \xi(t)=\xi(t s) \Delta(s)^{1 / 2}, \quad s, t \in G, \xi \in L^{2}(G) .
$$

The von Neumann algebra generated by $\lambda(G)$ is called the group von Neumann algebra of $G$ and is denoted by $V N(G)$. It follows that $W \in L^{\infty}(G) \bar{\otimes} V N(G)$ and $V \in V N(G)^{\prime} \bar{\otimes} L^{\infty}(G)$.

The set of coefficient functions of the left regular representation,

$$
A(G)=\left\{\psi: G \rightarrow \mathbb{C}: \psi(s)=\langle\lambda(s) \xi, \eta\rangle, \xi, \eta \in L^{2}(G), s \in G\right\},
$$

is called the Fourier algebra of $G$. It was shown by Eymard that, endowed with the norm

$$
\|\psi\|_{A(G)}=\inf \left\{\|\xi\|_{L^{2}(G)}\|\eta\|_{L^{2}(G)}: \psi(\cdot)=\langle\lambda(\cdot) \xi, \eta\rangle\right\}
$$

$A(G)$ is a Banach algebra under pointwise multiplication [15, Proposition 3.4]. Furthermore, it is the predual of $V N(G)$, where the duality is given by

$$
\langle\psi, \lambda(s)\rangle=u(s), \quad \psi \in A(G), s \in G .
$$

The inclusion $A(G) \subseteq V N(G)^{*}$ induces a canonical operator space structure on $A(G)$.

The adjoint of pointwise multiplication : : $A(G) \widehat{\otimes} A(G) \rightarrow A(G)$ defines a co-associative comultiplication $\widehat{\Gamma}: V N(G) \rightarrow V N(G) \bar{\otimes} V N(G)$ satisfying $\widehat{\Gamma}(\lambda(s))=\lambda(s) \otimes \lambda(s), s \in G$. There are left and right fundamental unitaries $\widehat{W}, \widehat{V} \in \mathcal{B}\left(L^{2}(G \times G)\right)$ which implement the co-multiplication via

$$
\widehat{\Gamma}(x)=\widehat{W}^{*}(1 \otimes x) \widehat{W}=\widehat{V}(x \otimes 1) \widehat{V}, \quad x \in V N(G) .
$$


They are given specifically by

$$
\widehat{W} \xi(s, t)=\xi(t s, t), \quad \widehat{V} \xi(s, t)=W \xi(s, t)=\xi\left(s, s^{-1} t\right), \quad s, t \in G, \xi \in L^{2}(G \times G) .
$$

A function $\varphi \in L^{\infty}(G)$ is a completely bounded multiplier of $A(G)$ if the map

$$
m_{\varphi}: A(G) \ni v \mapsto \varphi \cdot v \in A(G)
$$

is completely bounded. Under the natural norm, the space $M_{c b} A(G)$ of completely bounded multipliers becomes a completely contractive Banach algebra. It is known that $M_{c b} A(G)$ is a dual operator space with predual $Q_{c b}(G)$ [10, Proposition 1.10] (see also [24]). A locally compact group $G$ has the approximation property $(A P)$ if there exists a net $\left(v_{i}\right)$ in $A(G)$ such that $v_{i} \rightarrow 1$ $\sigma\left(M_{c b} A(G), Q_{c b}(G)\right)$. By [21, Remark 1.2], the net $\left(v_{i}\right)$ may always be chosen in $A(G) \cap C_{c}(G)$.

Given $\varphi \in M_{c b} A(G)$, the adjoint of $m_{\varphi}$ defines a normal completely bounded map on $V N(G)$. This map extends in a canonical fashion to a normal completely bounded $L^{\infty}(G)$-bimodule map $\widehat{\Theta}(\varphi)$ on $\mathcal{B}\left(L^{2}(G)\right)$. For example, when $\varphi \in A(G)$ we have

$$
\widehat{\Theta}(\varphi)(T)=(\varphi \otimes \mathrm{id}) \widehat{W}^{*}(1 \otimes T) \widehat{W}, \quad T \in \mathcal{B}\left(L^{2}(G)\right) .
$$

In [32] it was shown that the assignment $\varphi \mapsto \widehat{\Theta}(\varphi)$ is a completely isometric algebra isomorphism

$$
\widehat{\Theta}: M_{c b} A(G) \cong \mathcal{C B}_{L^{\infty}(G)}^{\sigma, V N(G)}\left(\mathcal{B}\left(L^{2}(G)\right)\right)
$$

from $M_{c b} A(G)$ onto the algebra $\mathcal{C B}_{L^{\infty}(G)}^{\sigma, V N(G)}\left(\mathcal{B}\left(L^{2}(G)\right)\right)$ of normal completely bounded $L^{\infty}(G)$ bimodule maps on $\mathcal{B}\left(L^{2}(G)\right)$ which leave $V N(G)$ globally invariant.

A $W^{*}$-dynamical system $(M, G, \alpha)$ consists of a von Neumann algebra $M \subseteq \mathcal{B}(H)$ endowed with a homomorphism $\alpha: G \rightarrow \operatorname{Aut}(M)$ such that for each $x \in M$, the map $G \ni s \rightarrow \alpha_{s}(x) \in M$ is weak* continuous. Every action induces a co-action of $L^{\infty}(G)$ on $M$, that is, a normal unital injective *-homomorphism $\alpha: M \rightarrow L^{\infty}(G) \bar{\otimes} M$ and a corresponding right $L^{1}(G)$-module structure on $M$ $[37, \S 18.6]$. At this level, the co-action is co-associative in the sense that $(\Gamma \otimes \mathrm{id}) \circ \alpha=(\mathrm{id} \otimes \alpha) \circ \alpha$, and the module structure is determined by

$$
x \star f=(f \otimes \mathrm{id}) \alpha(x), \quad f \in L^{1}(G), x \in M .
$$

Note that the predual $M_{*}$ becomes a left operator $L^{1}(G)$-module via $\alpha_{*}: L^{1}(G) \widehat{\otimes} M_{*} \rightarrow M_{*}$.

The crossed product of $M$ by $G$, denoted $G \bar{\rtimes}_{\alpha} M$, is the von Neumann subalgebra of $\mathcal{B}\left(L^{2}(G)\right) \bar{\otimes} M$ generated by $\alpha(M)$ and $V N(G) \otimes 1$. When $\alpha$ is clear from context, we often simply write $G \bar{\rtimes} M$. For $\mu \in M(G)$, we write $u(\mu)=\lambda(\mu) \otimes 1$ for the canonical image of $\lambda(\mu)$ in the crossed product. Recall that $\lambda$ is involutive, so that, in particular, $u(f)^{*}=u\left(f^{o}\right)$, where

$$
f^{o}(s)=\Delta(s)^{-1} \overline{f\left(s^{-1}\right)}, \quad f \in L^{1}(G) .
$$

Any action $\alpha$ admits a dual co-action $\widehat{\alpha}: G \bar{\rtimes} M \rightarrow V N(G) \bar{\otimes}(G \bar{\rtimes} M)$ of $V N(G)$ on the crossed product, given by

$$
\widehat{\alpha}(T)=\left(\widehat{W}^{*} \otimes 1\right)(1 \otimes T)(\widehat{W} \otimes 1), \quad T \in G \bar{\rtimes} M .
$$

On the generators we have $\widehat{\alpha}(\hat{x} \otimes 1)=\widehat{\Gamma}(\hat{x}) \otimes 1, \hat{x} \in V N(G)$ and $\widehat{\alpha}(\alpha(x))=1 \otimes \alpha(x), x \in M$. Moreover,

$$
(G \bar{\rtimes} M)^{\widehat{\alpha}}=\{T \in G \bar{\rtimes} M \mid \widehat{\alpha}(T)=1 \otimes T\}=\alpha(M) .
$$

This co-action yields a canonical right operator $A(G)$-module structure on the crossed product $G \bar{\rtimes} M$ via

$$
T \cdot \psi=(\psi \otimes \mathrm{id}) \widehat{\alpha}(T), \quad \psi \in A(G), T \in G \bar{\rtimes} M .
$$

It follows from equations (3) and (4) that

$$
T \cdot \psi=(\widehat{\Theta}(\psi) \otimes \mathrm{id})(T), \quad \psi \in A(G), T \in G \bar{\rtimes} M .
$$


Let $N=G \bar{\rtimes} M$, and let $\varphi: V N(G)^{+} \rightarrow[0, \infty]$ denote the Plancherel weight on $V N(G)$. Then

$$
E=(\varphi \otimes \mathrm{id}) \circ \widehat{\alpha}: N^{+} \rightarrow \widehat{N}^{+}
$$

is an operator-valued weight in the sense of [20], where $\widehat{N}^{+}$is the extended positive part of $N$ (i.e., the set of homogeneous, additive, lower semi-continuous functions on $N_{*}^{+}$). Following [26], let

$$
\mathcal{M}_{(\varphi \otimes \mathrm{id})}^{+}:=\left\{T \in(V N(G) \bar{\otimes} N)^{+} \mid \exists C_{T}>0 \varphi((\mathrm{id} \otimes \omega)(T)) \leq C_{T}\|\omega\|, \omega \in\left(N_{*}\right)_{+}\right\} .
$$

Also, let $\mathcal{N}_{(\varphi \otimes \mathrm{id})}:=\left\{T \in V N(G) \bar{\otimes} N \mid T^{*} T \in \mathcal{M}_{(\varphi \otimes \mathrm{id})}^{+}\right\}$, and $\mathcal{M}_{(\varphi \otimes \mathrm{id})}:=\operatorname{span} \mathcal{M}_{(\varphi \otimes \mathrm{id})}^{+}=$ $\mathcal{N}_{(\varphi \otimes \mathrm{id})}^{*} \mathcal{N}_{(\varphi \otimes \mathrm{id})}$. The operator-valued weight $E$ defines a linear map from the weak*-dense subspace $N_{1}:=\widehat{\alpha}^{-1}\left(\mathcal{M}_{(\varphi \otimes \mathrm{id})}\right)$ of $N$ to $N$ satisfying (see [26, $\S 2$, Corollary 1.3] or [19, 20] for details):

(1) $E\left(T^{*}\right)=E(T)^{*}, T \in N_{1}$,

(2) $E\left(T^{*} T\right) \geq 0, T \in N_{1}$,

(3) $E(x T y)=x E(T) y, T \in N_{1}, x, y \in M$,

(4) For $A, B \in N_{0}:=\widehat{\alpha}^{-1}\left(\mathcal{N}_{(\varphi \otimes \mathrm{id})}\right)$, the map $T \mapsto E\left(A^{*} T B\right)$ is $\sigma$-continuous on bounded sets.

By the proof of [26, Lemma 2.6], $\left\{u(f) T u(g) \mid T \in N, f, g \in C_{c}(G)\right\} \subseteq N_{1}$ and is weak* dense in $N$. Note that

$$
\begin{aligned}
\widehat{\alpha}(E(u(f) T u(g))) & =\widehat{\alpha}((\varphi \otimes \mathrm{id}) \widehat{\alpha}(u(f) T u(g))) \\
& =(\varphi \otimes \mathrm{id} \otimes \mathrm{id})((\mathrm{id} \otimes \widehat{\alpha})(\widehat{\alpha}(u(f) T u(g)))) \\
& =(\varphi \otimes \mathrm{id} \otimes \mathrm{id})((\widehat{\Gamma} \otimes \mathrm{id})(\widehat{\alpha}(u(f) T u(g)))) \\
& =1 \otimes(\varphi \otimes \mathrm{id})(\widehat{\alpha}(u(f) T u(g))),
\end{aligned}
$$

where the last line follows from strong right invariance of the Plancherel weight (see e.g., [25, Proposition 3.1]). It follows that $E$ maps $N_{1}$ into $\alpha(M) \cong M$, and we may view $E$ as an operator-valued weight from $N$ to $M$. When $G$ is discrete, the Plancherel weight $\varphi=\left\langle(\cdot) \delta_{e}, \delta_{e}\right\rangle$ and the operatorvalued weight $E$ becomes the canonical faithful normal conditional expectation $E: M \bar{\rtimes} G \rightarrow M$.

A $C^{*}$-dynamical system $(A, G, \alpha)$ consists of a $C^{*}$-algebra $A$ endowed with a homomorphism $\alpha: G \rightarrow \operatorname{Aut}(A)$ such that for each $a \in A$, the map $G \ni s \mapsto \alpha_{s}(a) \in A$ is norm continuous. A covariant representation $(\pi, \sigma)$ of $(A, G, \alpha)$ consists of a representation $\pi: A \rightarrow \mathcal{B}(H)$ and a unitary representation $\sigma: G \rightarrow \mathcal{B}(H)$ such that $\pi\left(\alpha_{s}(a)\right)=\sigma(s) \pi(a) \sigma(s)^{-1}$ for all $s \in G$. Given a covariant representation $(\pi, \sigma)$, we let

$$
(\pi \times \sigma)(f)=\int_{G} \pi(f(t)) \sigma(t) d t, \quad f \in C_{c}(G, A) .
$$

The full crossed product $G \rtimes_{f} A$ is the completion of $C_{c}(G, A)$ in the norm

$$
\|f\|=\sup _{(\pi, \sigma)}\|(\pi \times \sigma)(f)\|
$$

where the supremum is taken over all covariant representations $(\pi, \sigma)$ of $(A, G, \alpha)$.

Let $\pi_{u}: A \hookrightarrow \mathcal{B}\left(H_{u}\right)$ be the universal representation of $A$. Then $\left(\tilde{\pi}_{u}, u\right)$ is a covariant representation on $L^{2}\left(G, H_{u}\right)$, where

$$
\tilde{\pi}_{u}(a) \xi(t)=\pi_{u}\left(\alpha_{t^{-1}}(a)\right) \xi(t), \quad u(s) \xi(t)=\xi\left(s^{-1} t\right), \quad \xi \in L^{2}\left(G, H_{u}\right) .
$$

The reduced crossed product $G \rtimes A$ is defined to be the norm closure of $\tilde{\pi}_{u} \times u\left(C_{c}(G, A)\right)$.

Analogously to the von Neumann setting, one can view the action through the injective *homomorphism

$$
\alpha: A \ni a \mapsto\left(s \mapsto \alpha_{s^{-1}}(a)\right) \in C_{b}(G, A) \subseteq M\left(C_{0}(G) \otimes^{\vee} A\right),
$$

which, under the canonical inclusion $M\left(C_{0}(G) \otimes^{\vee} A\right) \subseteq L^{\infty}(G) \bar{\otimes} A^{* *}$ is nothing but the representation $\tilde{\pi}_{u}$ above. Moreover, $\left\langle\alpha(A)\left(C_{0}(G) \otimes 1\right)\right\rangle=C_{0}(G) \otimes \vee A$ and it follows that

$$
G \rtimes A=\left\langle\alpha(A)\left(C_{\lambda}^{*}(G) \otimes 1\right)\right\rangle \subseteq M\left(\mathcal{K}\left(L^{2}(G)\right) \otimes^{\vee} A\right) .
$$


Letting $B=G \rtimes A$ we define, as above,

$$
\mathcal{A}_{(\varphi \otimes \mathrm{id})}^{+}:=\left\{T \in M\left(C_{\lambda}^{*}(G) \otimes^{\vee} B\right)^{+} \mid \exists C_{T}>0 \varphi((\mathrm{id} \otimes \omega)(T)) \leq C_{T}\|\omega\|, \omega \in\left(B^{*}\right)_{+}\right\} .
$$

Also, let

$$
\mathcal{B}_{(\varphi \otimes \mathrm{id})}:=\left\{T \in M\left(C_{\lambda}^{*}(G) \otimes^{\vee} B\right) \mid T^{*} T \in \mathcal{M}_{(\varphi \otimes \mathrm{id})}^{+}\right\},
$$

and $\mathcal{A}_{(\varphi \otimes \mathrm{id})}:=\operatorname{span} \mathcal{A}_{(\varphi \otimes \mathrm{id})}^{+}=\mathcal{B}_{(\varphi \otimes \mathrm{id})}^{*} \mathcal{B}_{(\varphi \otimes \mathrm{id})}$. The dual co-action in this setting is

$$
\widehat{\alpha}: B \ni T \mapsto\left(\widehat{W}^{*} \otimes 1\right)(1 \otimes T)(\widehat{W} \otimes 1) \in M\left(C_{\lambda}^{*}(G) \otimes^{\vee} B\right),
$$

which extends by the above formula to a normal *-homomorphism $\widehat{\alpha}: B^{\prime \prime} \rightarrow V N(G) \bar{\otimes} B^{\prime \prime}$. The operator-valued weight $E=(\varphi \otimes$ id $) \circ \widehat{\alpha}: B^{\prime \prime} \rightarrow{\widehat{B^{\prime \prime}}}^{+}$restricts to a linear map from $B_{1}:=$ $\widehat{\alpha}^{-1}\left(\mathcal{A}_{(\varphi \otimes \mathrm{id})}\right)$ to $B$ satisfying $E(u(f) T u(g)) \in \alpha(A)$ for every $T \in B$ and $f, g \in C_{c}(G)$ (see $\left.[26, \S 3]\right)$.

\section{Fubini CROSSED PROduCts}

3.1. $W^{*}$-setting. Let $(M, G, \alpha)$ be a $W^{*}$-dynamical system. Viewing the crossed product $G \bar{\rtimes} M$ as a 'twisted' tensor product, it is natural to study a 'twisted' version of the normal Fubini tensor product.

Definition 3.1. Let $(M, G, \alpha)$ be a $W^{*}$-dynamical system. For a $G$-invariant weak* closed subspace $X \subseteq M$ we define the Fubini crossed product of $X$ by $G$ as

$$
G \bar{\rtimes}_{\mathcal{F}} X:=\left\{T \in G \bar{\rtimes} M \mid E(u(f) T u(g)) \in \alpha(X), f, g \in C_{c}(G)\right\} .
$$

Based on ideas in [23], a notion of Fubini crossed product for countable discrete groups acting on operator spaces was introduced in [40, Definition 4.1]. The natural analogue of [40, Definition 4.1] for locally compact groups acting on dual operator spaces is equivalent to our Definition 3.1, as we now show.

Proposition 3.2. Let $(M, G, \alpha)$ be a $W^{*}$-dynamical system. For any $G$-invariant weak* ${ }^{*}$ closed subspace $X \subseteq M$,

$$
G \bar{\rtimes}_{\mathcal{F}} X=\left\{T \in G \bar{\rtimes} M \mid(\rho \otimes \mathrm{id})(T) \in X, \rho \in \mathcal{T}\left(L^{2}(G)\right)\right\} .
$$

Proof. First consider $T \in G \bar{\rtimes} M$ of the form $\alpha(x) u(r), x \in M, r \in G$. Then for $f, g \in C_{c}(G)$ we have, on the one hand,

$$
\begin{aligned}
& E\left(u(f)^{*} T u(g)\right)=(\varphi \otimes \mathrm{id}) \widehat{\alpha}\left(u\left(f^{o}\right) \alpha(x) u(r) u(g)\right) \\
& =(\varphi \otimes \mathrm{id} \otimes \mathrm{id})\left(\iint f^{o}(s) g(t)(\lambda(s) \otimes \lambda(s) \otimes 1)(1 \otimes \alpha(x))(\lambda(r t) \otimes \lambda(r t) \otimes 1) d s d t\right) \\
& =(\varphi \otimes \mathrm{id} \otimes \mathrm{id})\left(\iint f^{o}(s) g(t)(1 \otimes u(s) \alpha(x) u(r t))(\lambda(s r t) \otimes 1 \otimes 1) d s d t\right) \\
& =\int f^{o}(s) g\left(r^{-1} s^{-1}\right) u(s) \alpha(x) u\left(s^{-1}\right) d s \\
& =\int \overline{f\left(s^{-1}\right)} g\left(r^{-1} s^{-1}\right) \Delta\left(s^{-1}\right)(1 \otimes \lambda(s)) \alpha(x)\left(1 \otimes \lambda\left(s^{-1}\right) d s .\right.
\end{aligned}
$$


Performing the substitution $s \mapsto s^{-1}$ yields

$$
\begin{aligned}
E\left(u(f)^{*} T u(g)\right) & =\int \overline{f(s)} g\left(r^{-1} s\right)\left(\lambda\left(s^{-1}\right) \otimes 1\right) \alpha(x)(\lambda(s) \otimes 1) d s \\
& =(\bar{f} \cdot \lambda(r) g \otimes \mathrm{id} \otimes \mathrm{id})(\Gamma \otimes \mathrm{id})(\alpha(x)) \\
& =(\bar{f} \cdot \lambda(r) g \otimes \mathrm{id} \otimes \mathrm{id})(\mathrm{id} \otimes \alpha)(\alpha(x)) \\
& =\alpha(x \star(\bar{f} \cdot \lambda(r) g)) .
\end{aligned}
$$

On the other hand, $\left.\lambda(r) \cdot \omega_{g, f}\right|_{L^{\infty}(G)}=\bar{f} \cdot \lambda(r) g \in L^{1}(G)$, so that

$$
\left(\omega_{g, f} \otimes \mathrm{id}\right)(\alpha(x) u(r))=\left(\left.\lambda(r) \cdot \omega_{g, f}\right|_{L^{\infty}(G)} \otimes \mathrm{id}\right)(\alpha(x))=x \star(\bar{f} \cdot \lambda(r) g) .
$$

Thus,

$$
\alpha\left(\left(\omega_{g, f} \otimes \mathrm{id}\right)(T)\right)=E\left(u(f)^{*} T u(g)\right) .
$$

By normality the above equality is valid for every $T \in G \bar{\rtimes} M$. Since $\operatorname{span}\left\{\omega_{g, f} \mid f, g \in C_{c}(G)\right\}$ is dense in $\mathcal{T}\left(L^{2}(G)\right)$, the claim is established.

The following example further justifies the terminology of Fubini crossed products.

Example 3.3. Let $G$ be a locally compact group acting trivially on a von Neumann algebra $M$. Then $G \bar{\rtimes} M=V N(G) \bar{\otimes} M$. By Proposition 3.2, for any $G$-invariant weak* closed subspace $X \subseteq M$ we have

$$
G \bar{\rtimes}_{\mathcal{F}} X=\left\{T \in V N(G) \bar{\otimes} M \mid(\rho \otimes \mathrm{id})(T) \in X, \rho \in \mathcal{T}\left(L^{2}(G)\right)\right\}=V N(G) \bar{\otimes}_{\mathcal{F}} X .
$$

Proposition 3.4. Let $(M, G, \alpha)$ be a $W^{*}$-dynamical system. For any $G$-invariant weak* closed subspace $X \subseteq M$ we have

where $G \bar{\rtimes} X=\overline{\langle\alpha(X)(V N(G) \otimes 1)\rangle} w^{*}$.

$$
G \bar{\rtimes} X \subseteq G \bar{\rtimes}_{\mathcal{F}} X
$$

Proof. First consider $T \in G \bar{\rtimes} X$ of the form $\alpha(x) u(r), x \in X, r \in G$. Then, as shown in the proof of Proposition 3.2, for $f, g \in C_{c}(G)$ we have

$$
E\left(u(f)^{*} T u(g)\right)=\alpha(x \star(\bar{f} \cdot \lambda(r) g)),
$$

which belongs to $\alpha(X)$ by $L^{1}(G)$-invariance of $X$. The normality of $E\left(u(f)^{*}(\cdot) u(g)\right)$ ensures the same is true for arbitrary $T \in G \bar{\rtimes} X$.

Although Proposition 3.2 shows that Fubini crossed products are determined by the restriction of slice maps to the crossed product, our (equivalent) Definition 3.1 was motivated by considering the twisted slice map conditions

$$
E(u(f) T u(g))=(\varphi \otimes \mathrm{id} \otimes \mathrm{id})(\widehat{\Gamma} \otimes \mathrm{id})(u(f) T u(g)) \in \alpha(X), \quad f, g \in C_{c}(G),
$$

with the perspective that the Fubini crossed product is a twisted version of the Fubini tensor product. For discrete actions, the twisted slice map conditions are directly related to the $A(G)$ action on the crossed product.

Example 3.5. For any discrete $W^{*}$-dynamical system $(M, G, \alpha)$ and any $G$-invariant weak* closed subspace $X \subseteq M$, we have

$$
G \bar{\rtimes}_{\mathcal{F}} X=\{T \in G \bar{\rtimes} M \mid T \cdot \psi \in G \bar{\rtimes} X, \psi \in A(G)\} .
$$

First, observe that

$$
\begin{aligned}
\left\langle\delta_{s}, \lambda(f)\right\rangle & =f(s)=\left(\delta_{s^{-1}} * f\right)(e)=\left\langle\varphi, \lambda\left(\delta_{s^{-1}} * f\right)\right\rangle \\
& =\left\langle\varphi, \lambda\left(s^{-1}\right) \lambda(f)\right\rangle=\left\langle\varphi, \lambda(f) \lambda\left(s^{-1}\right)\right\rangle \\
& =\left\langle\lambda\left(s^{-1}\right) \cdot \varphi, \lambda(f)\right\rangle
\end{aligned}
$$


for all $f \in L^{1}(G)$. Thus, $\delta_{s}=\lambda\left(s^{-1}\right) \cdot \varphi$ as elements of $A(G)$, and we have

$$
\begin{aligned}
T \cdot \delta_{s} & =\left(\lambda\left(s^{-1}\right) \cdot \varphi \otimes \mathrm{id} \otimes \mathrm{id}\right) \widehat{\alpha}(T) \\
& =(\varphi \otimes \mathrm{id} \otimes \mathrm{id})\left(\widehat{\alpha}(T)\left(\lambda\left(s^{-1}\right) \otimes 1 \otimes 1\right)\right) \\
& =(\varphi \otimes \mathrm{id} \otimes \mathrm{id})\left(\widehat{\alpha}(T)\left(\lambda\left(s^{-1}\right) \otimes \lambda\left(s^{-1}\right) \otimes 1\right)(1 \otimes \lambda(s) \otimes 1)\right) \\
& =(\varphi \otimes \mathrm{id} \otimes \mathrm{id})\left(\widehat{\alpha}\left(T u\left(s^{-1}\right)\right)(1 \otimes \lambda(s) \otimes 1)\right) \\
& =(\varphi \otimes \mathrm{id} \otimes \mathrm{id})\left(\widehat{\alpha}\left(T u\left(s^{-1}\right)\right)\right) u(s) \\
& =E\left(T u\left(s^{-1}\right)\right) u(s), \quad s \in G .
\end{aligned}
$$

Suppose that $T \cdot \psi \in G \bar{\rtimes} X$ for all $\psi \in A(G)$, so that $E\left(T u\left(s^{-1}\right)\right) u(s) \in G \bar{\rtimes} X$ for all $s \in G$. Since $E(G \bar{\rtimes} X) \subseteq \alpha(X)$ we see that

$$
\begin{aligned}
E\left(T u\left(s^{-1}\right)\right) & =E\left(E\left(T u\left(s^{-1}\right)\right)\right)=E\left(E\left(T u\left(s^{-1}\right)\right) u(s) u\left(s^{-1}\right)\right) \\
& =E\left(\left(T \cdot \delta_{s}\right) u\left(s^{-1}\right)\right) \in E(G \bar{\rtimes} X) \subseteq \alpha(X), \quad s \in G .
\end{aligned}
$$

By $G$-equivariance of $E$ it follows that

$$
\alpha_{t}(E(T u(s)))=E\left(u(t) T u\left(s t^{-1}\right)\right) \in \alpha(X)
$$

for all $s, t \in G$, which implies $E(u(f) T u(g)) \in \alpha(X)$ for all $f, g \in C_{c}(G)$, i.e., $T \in G \bar{\rtimes}_{\mathcal{F}} X$.

Conversely, if $T \in G \bar{\rtimes}_{\mathcal{F}} X$, then by discreteness $E\left(T u\left(s^{-1}\right)\right) \in \alpha(X)$ so that $E\left(T u\left(s^{-1}\right)\right) u(s)=$ $T \cdot \delta_{s} \in G \bar{\rtimes} X$ for all $s \in G$. Norm density of $C_{c}(G) \cap A(G)$ in $A(G)$ then implies $T \cdot \psi \in G \bar{\rtimes} X$ for all $\psi \in A(G)$.

Note that in proving (5) we have also shown

$$
G \bar{\rtimes}_{\mathcal{F}} X=\left\{T \in G \bar{\rtimes} M \mid E\left(T u\left(s^{-1}\right)\right) \in \alpha(X), s \in G\right\} .
$$

The von Neumann algebra analogue of [38, Proposition 3.4], which holds by [38, Remark 3.6], shows that for a discrete group $G$ with the AP acting on a von Neumann algebra $M$, for any $G$-invariant weak* closed subspace $X$, an element $T \in G \bar{\rtimes} M$ satisfying $E\left(T u\left(s^{-1}\right)\right) \in \alpha(X)$ for all $s \in G$ is necessarily contained in $G \bar{\rtimes} X$. Example 3.5 allows us to interpret this result as a "slice map property" with respect to the Fubini crossed product.

Definition 3.6. A $W^{*}$-dynamical system $(M, G, \alpha)$ has the slice map property if $G \bar{\rtimes} X=G \bar{\rtimes}{ }_{\mathcal{F}} X$ for every $G$-invariant weak* closed subspace $X \subseteq M$.

Example 3.7. Let $G$ be a locally compact group with the AP. As we shall see, by Corollary 4.8 below, every $W^{*}$-dynamical system $(M, G, \alpha)$ has the slice map property.

After the first version of this paper appeared, this result was obtained using different techniques by Andreou [1, Proposition 4.3].

Example 3.8. Let $G$ be a locally compact group acting trivially on $\mathcal{B}(H)$ for a separable Hilbert space $H$. By Example 3.3 we have $G \bar{\rtimes}_{\mathcal{F}} X=V N(G) \bar{\otimes}_{\mathcal{F}} X$ for any weak* closed subspace of $X$ of $\mathcal{B}(H)$. Hence, the trivial action has the slice map property if and only if $V N(G)$ has the dual slice map property for weak* closed subspaces of $\mathcal{B}(H)$, equivalently, $V N(G)$ has the w*OAP. If, in addition, $G$ is inner amenable in the sense of Paterson [33, 2.35H] (e.g., $G$ is discrete), then it follows from $[9$, Corollary 4.8] that $G$ has the AP.

3.2. $C^{*}$-setting. We have the analogous notions in the setting of $C^{*}$-dynamical systems.

Definition 3.9. Let $(A, G, \alpha)$ be a $C^{*}$-dynamical system. For a $G$-invariant closed subspace $X \subseteq A$ we define the Fubini crossed product of $X$ by $G$ as

$$
G \rtimes_{\mathcal{F}} X:=\left\{T \in G \rtimes A \mid E(u(f) T u(g)) \in \alpha(X), f, g \in C_{c}(G)\right\} .
$$


Proposition 3.10. Let $(A, G, \alpha)$ be a $C^{*}$-dynamical system. For any $G$-invariant closed subspace $X \subseteq A$,

$$
G \rtimes_{\mathcal{F}} X=\left\{T \in G \rtimes A \mid(\rho \otimes \mathrm{id})(T) \in X, \rho \in \mathcal{T}\left(L^{2}(G)\right)\right\} .
$$

Proof. First consider $T \in G \rtimes A$ of the form $\alpha(x) u(h), x \in A, h \in C_{c}(G)$. For $f, g \in C_{c}(G)$, the exact same integral calculation from the proof of Proposition 3.2 shows that

$$
E\left(u(f)^{*} T u(g)\right)=\alpha(x \star(\bar{f} \cdot(h * g))),
$$

where $h * g$ is the convolution of $h$ and $g$ in $L^{1}(G)$. Since $\left.\lambda(h) \cdot \omega_{g, f}\right|_{L^{\infty}(G)}=\bar{f} \cdot(h * g) \in L^{1}(G)$, we have

$$
\left(\omega_{g, f} \otimes \mathrm{id}\right)(\alpha(x) u(h))=\left(\left.\lambda(h) \cdot \omega_{g, f}\right|_{L^{\infty}(G)} \otimes \mathrm{id}\right)(\alpha(x))=x \star(\bar{f} \cdot(h * g)) .
$$

Thus,

$$
\alpha\left(\left(\omega_{g, f} \otimes \mathrm{id}\right)(T)\right)=E\left(u(f)^{*} T u(g)\right) .
$$

Since the left hand side is norm continuous in $T$, and the right hand side is weak* continuous for $T$ in bounded sets (viewing $E$ as an operator-valued weight on $\left.(G \rtimes A)^{\prime \prime}\right)$, the above equality is valid for every $T \in G \rtimes A$, establishing the claim as in Proposition 3.2.

Proposition 3.11. Let $(A, G, \alpha)$ be a $C^{*}$-dynamical system. For any $G$-invariant closed subspace $X \subseteq A$ we have

where $G \rtimes X={\overline{\left\langle\alpha(X)\left(C_{\lambda}^{*}(G) \otimes 1\right)\right.}}^{\|\cdot\|}$.

$$
G \rtimes X \subseteq G \rtimes_{\mathcal{F}} X
$$

Proof. By the proof of Proposition 3.10, for every $f, g \in C_{c}(G)$,

$$
\alpha\left(\left(\omega_{g, f} \otimes \mathrm{id}\right)(T)\right)=E\left(u(f)^{*} T u(g)\right), \quad T \in G \rtimes A .
$$

In particular, $E\left(u(f)^{*}(\cdot) u(g)\right)$ is norm continuous in $T$, and for $T=\alpha(x) u(h), x \in X, h \in C_{c}(G)$,

$$
E\left(u(f)^{*} T u(g)\right)=\alpha(x \star(\bar{f} \cdot(h * g))) \in \alpha(X)
$$

by $L^{1}(G)$-invariance of $X$. The norm continuity of $E\left(u(f)^{*}(\cdot) u(g)\right)$ ensures the same is true for arbitrary $T \in G \rtimes X$.

Definition 3.12. Let $(A, G, \alpha)$ be a $C^{*}$-dynamical system. We say that the action $\alpha$ has the slice map property if $G \rtimes X=G \rtimes_{\mathcal{F}} X$ for every $G$-invariant closed subspace $X \subseteq A$.

Example 3.13. Let $G$ be a locally compact group with the AP. As we shall see, by Corollary 4.11 below, every $C^{*}$-dynamical system $(A, G, \alpha)$ has the slice map property.

Following [35, Definition 1.5], a $C^{*}$-dynamical system $(A, G, \alpha)$ is exact if for every $G$-invariant (norm-closed two-sided) ideal $I \triangleleft A$ the sequence

$$
0 \rightarrow G \rtimes I \hookrightarrow G \rtimes A \rightarrow G \rtimes A / I \rightarrow 0
$$

is exact. A locally compact group $G$ is exact if every $C^{*}$-dynamical system $(A, G, \alpha)$ is exact [23].

Proposition 3.14. Let $(A, G, \alpha)$ be a $C^{*}$-dynamical system. If $(A, G, \alpha)$ has the slice map property then it is exact.

Proof. We follow similar lines to [35, Proposition 1.6]. Let $I \triangleleft A$ be a $G$-invariant ideal, and let $q: A \rightarrow A / I$ denote the quotient map. We show that $J:=\operatorname{Ker}(\operatorname{id} \rtimes q) \subseteq G \rtimes I$.

Fix $f, g \in C_{c}(G)$ and let $E_{f, g}^{A}:=\alpha_{A}^{-1}\left(E^{A}(u(f)(\cdot) u(g))\right)$ and $E_{f, g}^{A / I}:=\alpha_{A / I}^{-1}\left(E^{A / I}(u(f)(\cdot) u(g))\right)$. Then $E_{f, g}^{A}: G \rtimes A \rightarrow A$, and by the proof of Proposition 3.10

$$
E_{f, g}^{A}(T)=\alpha_{A}^{-1}\left(E^{A}\left(u\left(f^{o}\right)^{*} T u(g)\right)\right)=\left(\omega_{g, f^{\circ}} \otimes \mathrm{id}\right)(T), \quad T \in G \rtimes A .
$$


Similarly for $E_{f, g}^{A / I}$. Hence, for any $T \in G \rtimes A$,

$$
E_{f, g}^{A / I}((\mathrm{id} \rtimes q)(T))=\left(\omega_{g, f^{\circ}} \otimes \mathrm{id}\right)((\mathrm{id} \rtimes q)(T))=q\left(\left(\omega_{g, f^{\circ}} \otimes \mathrm{id}\right)(T)\right)=q \circ E_{f, g}^{A}(T) .
$$

Thus, $E_{f, g}^{A}(J) \subseteq \operatorname{Ker}(q)=I$. Since $f$ and $g$ in $C_{c}(G)$ were arbitrary and the action has the slice map property,

$$
J \subseteq G \rtimes_{\mathcal{F}} I=G \rtimes I .
$$

\section{Fejér Representations in Crossed Products}

We now establish our Fejér representation for arbitrary elements in $C^{*}$ - and von Neumann crossed products by locally compact groups with the AP. Throughout the proof we adopt the standard leg notation for fundamental unitaries, e.g., $W_{12}=(W \otimes 1), W_{23}=(1 \otimes W)$, etc.

Theorem 4.1. Let $G$ be a locally compact group, and denote by $\left(f_{i}\right) \subseteq C_{c}(G)_{\|\cdot\|_{1}=1}^{+}$a symmetric bounded approximate identity for $L^{1}(G)$. Then the following are equivalent.

(1) G has the AP.

(2) There exists a net $\left(h_{j}\right)$ in $A(G) \cap C_{c}(G)$ such that for every $W^{*}$-dynamical system $(M, G, \alpha)$,

$$
T=w^{*}-\lim _{j} \lim _{i} \int_{G} \frac{h_{j}(x)}{\Delta(x)} E\left(u\left(f_{i}\right) T u\left(f_{i}\right) u\left(x^{-1}\right)\right) u(x) d x, \quad T \in G \bar{\rtimes} M .
$$

Remark 4.2. If $G$ is weakly amenable, then the net $\left(h_{i}\right)$ can be chosen bounded in the $M_{c b} A(G)$ norm, and if $G$ is amenable, bounded in the $A(G)$-norm.

Remark 4.3. When $G$ is discrete, putting $f_{i}=\delta_{e}$, the Fejér representation (6) simplifies to

$$
T=w^{*}-\lim _{j} \sum_{x \in G} h_{j}(x) E\left(T u\left(x^{-1}\right)\right) u(x) .
$$

The first step in the proof of Theorem 4.1 is based on Lemma 4.4 below, which in turn is a version of [26, Lemma 2.9].

Lemma 4.4. Let $(M, G, \alpha)$ be a $W^{*}$-dynamical system. For $T \in G \bar{\rtimes} M, f, g, h \in C_{c}(G)$, and $\xi, \eta \in L^{2}(G) \otimes H$, we have

$$
\int_{G} h(x)\left\langle E\left(u(f) T u(g) u\left(x^{-1}\right)\right) u(x) \xi, \eta\right\rangle=\langle\widehat{\alpha}(u(f) T)(W \otimes 1)(f \otimes \xi), \Delta \cdot \bar{h} \otimes \eta\rangle .
$$

Consequently,

$$
\int_{G} h(x) E\left(u(f) T u(g) u\left(x^{-1}\right)\right) u(x) d x=\left(\omega_{f, \Delta \cdot \bar{h}} \otimes \mathrm{id}\right)(\widehat{\alpha}(u(f) T)(W \otimes 1)) \in \mathcal{B}\left(L^{2}(G) \otimes H\right) .
$$


Proof. First consider the case when $T=\alpha(m) u(y)$ for some $m \in M$ and $y \in G$. Then for each $x \in G$,

$$
\begin{aligned}
& \left\langle E\left(u(f) T u(g) u\left(x^{-1}\right)\right) u(x) \xi, \eta\right\rangle=\left\langle(\varphi \otimes \mathrm{id} \otimes \mathrm{id})\left(\widehat{\alpha}\left(u(f) T u(g) u\left(x^{-1}\right)\right)(1 \otimes u(x))\right) \xi, \eta\right\rangle \\
& =\left\langle(\varphi \otimes \mathrm{id} \otimes \mathrm{id})\left(\widehat{\alpha}(u(f) T u(g))\left(\lambda\left(x^{-1}\right) \otimes 1 \otimes 1\right)\right) \xi, \eta\right\rangle \\
& =\left\langle(\varphi \otimes \mathrm{id} \otimes \mathrm{id})\left(\iint f(s) g(t)(\lambda(s) \otimes u(s)) \widehat{\alpha}(T)\left(\lambda\left(t x^{-1}\right) \otimes u(t)\right) d s d t\right) \xi, \eta\right\rangle \\
& =\left\langle(\varphi \otimes \mathrm{id} \otimes \mathrm{id})\left(\iint f(s) g(t)(1 \otimes u(s) \alpha(m) u(y t))\left(\lambda\left(s y t x^{-1}\right) \otimes 1 \otimes 1\right) d s d t\right) \xi, \eta\right\rangle \\
& =\varphi\left(\iint f(s) g(t)\langle u(s) \alpha(m) u(y t) \xi, \eta\rangle \lambda\left(s y t x^{-1}\right) d s d t\right) \\
& =\Delta(x) \varphi\left(\iint f(s) g\left(y^{-1} s^{-1} t x\right)\left\langle u(s) \alpha(m) u\left(s^{-1} t x\right) \xi, \eta\right\rangle \lambda(t) d s d t\right) \\
& =\Delta(x) \int f(s) g\left(y^{-1} s^{-1} x\right)\left\langle u(s) \alpha(m) u\left(s^{-1} x\right) \xi, \eta\right\rangle d s \\
& =\Delta(x) \int f(s) g\left(y^{-1} s^{-1} x\right)\left\langle u(s) T u\left(y^{-1} s^{-1} x\right) \xi, \eta\right\rangle d s .
\end{aligned}
$$

Thus,

$$
\begin{aligned}
& \int h(x)\left\langle E\left(u(f) T u(g) u\left(x^{-1}\right)\right) u(x) \xi, \eta\right\rangle d x \\
= & \iint \Delta \cdot h(x) f(s) g\left(y^{-1} s^{-1} x\right)\left\langle u(s) T u\left(y^{-1} s^{-1} x\right) \xi, \eta\right\rangle d s d x \\
= & \iiint \Delta \cdot h(x) f(s) g\left(y^{-1} s^{-1} x\right)\left\langle\left(u\left(y^{-1} s^{-1} x\right) \xi\right)(t),\left(T^{*} u(s)^{*} \eta\right)(t)\right\rangle d t d s d x \\
= & \iiint f(s)\left\langle W_{12}(g \otimes \xi)\left(y^{-1} s^{-1} x, t\right),\left(1 \otimes T^{*} u(s)^{*}\right)(\Delta \cdot \bar{h} \otimes \eta)(x, t)\right\rangle d t d s d x \\
= & \iiint f(s)\left\langle(\lambda(s y) \otimes 1 \otimes 1) W_{12}(g \otimes \xi)(x, t),\left(1 \otimes T^{*} u(s)^{*}\right)(\Delta \cdot \bar{h} \otimes \eta)(x, t)\right\rangle d t d s d x \\
= & \int f(s)\left\langle(\lambda(s y) \otimes 1 \otimes 1) W_{12}(g \otimes \xi),\left(1 \otimes T^{*} u(s)^{*}\right)(\Delta \cdot \bar{h} \otimes \eta)\right\rangle d s \\
= & \int f(s)\left\langle(\lambda(s y) \otimes u(s) T) W_{12}(g \otimes \xi), \Delta \cdot \bar{h} \otimes \eta\right\rangle d s \\
= & \int f(s)\left\langle\widehat{\alpha}(u(s) T) W_{12}(g \otimes \xi), \Delta \cdot \bar{h} \otimes \eta\right\rangle d s \\
= & \left\langle\widehat{\alpha}(u(f) T) W_{12}(g \otimes \xi), \Delta \cdot \bar{h} \otimes \eta\right\rangle .
\end{aligned}
$$

By norm continuity of $E(u(f)(\cdot) u(g))$, the result holds for $T$ belonging to $C^{*}(\alpha(M), u(G))$, and then an application of Kaplansky's density theorem together with the weak* continuity of $E(u(f)(\cdot) u(g))$ on bounded sets establishes the result for arbitrary $T \in G \bar{\rtimes} M$.

The next step in the proof of Theorem 4.1 is to reinterpret the Hilbert space inner product in (7) as a particular operator space duality which behaves well as we let $f$ and $h$ vary accordingly. To this end, we consider the following maps on $L^{\infty}(G) \otimes L^{2}(G, H)$ induced from the fundamental unitary $\widehat{W}$ :

$$
\Phi_{1}(f \otimes \xi)(s, t)=f(t s) \xi(t)=\Sigma \Gamma(f)(s, t) \xi(t), \quad s, t \in G,
$$

and

$$
\Phi_{2}(f \otimes \xi)(s, t)=f\left(t^{-1} s\right) \xi(t)=(\mathrm{id} \otimes \kappa) \Sigma \Gamma(f)(s, t) \xi(t), \quad s, t \in G,
$$


where $\kappa(f)(s)=f\left(s^{-1}\right)$ is the co-involution on $L^{\infty}(G)$. Note that on $C_{c}(G) \otimes L^{2}(G, H)$, the maps $\Phi_{1}$ and $\Phi_{2}$ coincide with $(\widehat{W} \otimes 1)$ and $\left(\widehat{W}^{*} \otimes 1\right)$, respectively.

Lemma 4.5. Let $G$ be a locally compact group and $H$ be a Hilbert space. Then $\Phi_{1}$ induces a complete contraction

$$
\Phi_{1}: M_{c b} A(G) \widehat{\otimes} L^{2}(G, H)_{c} \rightarrow L^{\infty}(G) \widehat{\otimes} L^{2}(G, H)_{c},
$$

where $L^{2}(G, H)_{c}$ refers to the column operator space structure on the Hilbert space tensor product $L^{2}(G) \otimes H$.

Proof. It is well-known that $\Sigma \Gamma: M_{c b} A(G) \rightarrow L^{\infty}(G) \otimes^{w^{*} h} L^{\infty}(G)$ is a complete isometry (see [36, Corollary 5.5] for the case of $\Gamma)$. Let ev : $L^{\infty}(G) \otimes\left(L^{2}(G) \otimes H\right) \rightarrow L^{2}(G) \otimes H$ be the evaluation map $(f, \xi) \mapsto\left(M_{f} \otimes 1\right) \xi$. Then ev extends to a complete contraction $L^{\infty}(G) \otimes{ }^{w^{*} h} L^{2}(G, H)_{c} \rightarrow L^{2}(G, H)_{c}$. Indeed, it is given by the following composition

$$
\begin{aligned}
L^{\infty}(G) \otimes^{w^{*} h} L^{2}(G, H)_{c} & \subseteq\left(\mathcal{B}\left(L^{2}(G)\right) \otimes 1_{H}\right) \otimes^{w^{*} h} L^{2}(G, H)_{c} \subseteq \mathcal{B}\left(L^{2}(G) \otimes H\right) \otimes^{w^{*} h} L^{2}(G, H)_{c} \\
& =\left(L^{2}(G, H)_{c} \otimes^{w^{*} h} L^{2}(G, H)_{c}^{*}\right) \otimes^{w^{*} h} L^{2}(G, H)_{c} \\
& =L^{2}(G, H)_{c} \otimes^{w^{*} h}\left(L^{2}(G, H)_{c}^{*} \otimes^{w^{*} h} L^{2}(G, H)_{c}\right) \\
& \cong L^{2}(G, H)_{c} \otimes^{w^{*} h}\left(L^{2}(G, H)_{c}^{*} \otimes^{h} L^{2}(G, H)_{c}\right) \quad \text { by [6, Corollary 3.5] } \\
& \cong L^{2}(G, H)_{c} \otimes^{w^{*} h}\left(L^{2}(G, H)_{c}^{*} \widehat{\otimes} L^{2}(G, H)_{c}\right) \quad \text { by [12, Proposition 9.3.2] } \\
& \rightarrow L^{2}(G, H)_{c},
\end{aligned}
$$

where in the last line we apply the dual pairing $L^{2}(G, H)_{c}^{*} \widehat{\otimes} L^{2}(G, H)_{c} \rightarrow \mathbb{C}$. By [6, Proposition $3.7]$ it follows that

$$
\left(\operatorname{id}_{L^{\infty}(G)} \otimes \mathrm{ev}\right): L^{\infty}(G) \otimes^{w^{*} h}\left(L^{\infty}(G) \otimes^{w^{*} h} L^{2}(G, H)_{c}\right) \rightarrow L^{\infty}(G) \otimes^{w^{*} h} L^{2}(G, H)_{c}
$$

is completely contractive, and hence, by the canonical identifications used above,

$$
\left(\operatorname{id}_{L^{\infty}(G)} \otimes \mathrm{ev}\right): L^{\infty}(G) \otimes^{w^{*} h}\left(L^{\infty}(G) \otimes^{w^{*} h} L^{2}(G)_{c}\right) \rightarrow L^{\infty}(G) \widehat{\otimes} L^{2}(G, H)_{c}
$$

is completely contractive. Since $M_{c b} A(G)$ is a dual operator space, we have

$$
M_{c b} A(G) \otimes^{w^{*} h} L^{2}(G, H)_{c} \cong M_{c b} A(G) \otimes^{h} L^{2}(G, H)_{c} \cong M_{c b} A(G) \widehat{\otimes} L^{2}(G, H)_{c}
$$

completely isometrically, appealing to [6, Corollary 3.5] and [12, Proposition 9.3.2] once again. Hence, [6, Proposition 3.7] entails that

$$
\left(\Sigma \Gamma \otimes \operatorname{id}_{L^{2}(G, H)_{c}}\right): M_{c b} A(G) \widehat{\otimes} L^{2}(G, H)_{c} \rightarrow L^{\infty}(G) \otimes^{w^{*} h}\left(L^{\infty}(G) \otimes^{w^{*} h} L^{2}(G, H)_{c}\right)
$$

is completely contractive. From (8) it follows that

$$
\Phi_{1}=\left(\operatorname{id}_{L^{\infty}(G)} \otimes \mathrm{ev}\right) \circ\left(\Sigma \Gamma \otimes \operatorname{id}_{L^{2}(G, H)_{c}}\right)
$$

on $M_{c b} A(G) \otimes L^{2}(G, H)_{c}$. It therefore extends to a complete contraction

$$
M_{c b} A(G) \widehat{\otimes} L^{2}(G, H)_{c} \rightarrow L^{\infty}(G) \widehat{\otimes} L^{2}(G, H)_{c} .
$$

Lemma 4.6. Let $G$ be a locally compact group and $H$ be a Hilbert space. Then $\Phi_{2}$ induces a complete contraction

$$
\Phi_{2}: L^{\infty}(G) \widehat{\otimes} L^{2}(G, H)_{c} \rightarrow L^{\infty}(G) \bar{\otimes} L^{2}(G, H)_{c} .
$$

Proof. Let $\pi_{1}: L^{\infty}(G) \rightarrow \mathcal{C B}\left(L^{\infty}(G)\right)$ and $\pi_{2}: L^{\infty}(G) \rightarrow \mathcal{C B}\left(L^{2}(G, H)_{c}\right)$ be the representations given by left multiplication, where the latter maps $f \mapsto M_{f} \otimes 1$. Since $\pi_{1}$ and $\pi_{2}$ are both weak*weak* continuous, the map $\pi_{1} \otimes \pi_{2}$ extends to a complete contraction

$$
\pi_{1} \otimes \pi_{2}: L^{\infty}(G) \bar{\otimes} L^{\infty}(G) \rightarrow \mathcal{C B}\left(L^{\infty}(G)\right) \bar{\otimes} \mathcal{C B}\left(L^{2}(G, H)_{c}\right) .
$$


Since $L^{\infty}(G) \widehat{\otimes} L^{1}(G)$ has the OAP, its dual $\mathcal{C B}\left(L^{\infty}(G)\right)=\left(L^{\infty}(G) \widehat{\otimes} L^{1}(G)\right)^{*}$ has the dual slice map property so that

$$
\begin{aligned}
\mathcal{C B}\left(L^{\infty}(G)\right) \bar{\otimes} \mathcal{C B}\left(L^{2}(G, H)_{c}\right) & =\left(L^{\infty}(G) \widehat{\otimes} L^{1}(G)\right)^{*} \bar{\otimes}\left(L^{2}(G, H)_{c} \widehat{\otimes} L^{2}(G, H)_{c}^{*}\right)^{*} \\
& =\left(\left(L^{\infty}(G) \widehat{\otimes} L^{1}(G)\right) \widehat{\otimes}\left(L^{2}(G, H)_{c} \widehat{\otimes} L^{2}(G, H)_{c}^{*}\right)\right)^{*} \\
& =\left(\left(L^{\infty}(G) \widehat{\otimes} L^{2}(G, H)_{c}\right) \widehat{\otimes}\left(L^{1}(G) \widehat{\otimes} L^{2}(G, H)_{c}^{*}\right)\right)^{*} \\
& =\mathcal{C} \mathcal{B}\left(L^{\infty}(G) \widehat{\otimes} L^{2}(G, H)_{c}, L^{\infty}(G) \bar{\otimes} L^{2}(G, H)_{c}\right) .
\end{aligned}
$$

By universality of the operator space projective tensor product it follows that $\pi_{1} \otimes \pi_{2}$ induces a complete contraction

$$
m:\left(L^{\infty}(G) \bar{\otimes} L^{\infty}(G)\right) \widehat{\otimes}\left(L^{\infty}(G) \widehat{\otimes} L^{2}(G, H)_{c}\right) \rightarrow L^{\infty}(G) \bar{\otimes} L^{2}(G, H)_{c} .
$$

By equation (9), we see that

$$
\Phi_{2}=m \circ\left((\mathrm{id} \otimes \kappa) \Sigma \Gamma \otimes \mathrm{id}_{L^{\infty}(G) \bar{\otimes} L^{2}(G, H)_{c}}\right) \circ i
$$

on $L^{\infty}(G) \otimes L^{2}(G, H)_{c}$, where

$$
i: L^{\infty}(G) \widehat{\otimes} L^{2}(G, H)_{c} \ni f \otimes \eta \mapsto f \otimes(1 \otimes \eta) \in L^{\infty}(G) \widehat{\otimes}\left(L^{\infty}(G) \bar{\otimes} L^{2}(G, H)_{c}\right)
$$

and

$((\mathrm{id} \otimes \kappa) \Sigma \Gamma \otimes \mathrm{id}): L^{\infty}(G) \widehat{\otimes}\left(L^{\infty}(G) \bar{\otimes} L^{2}(G, H)_{c}\right) \rightarrow\left(L^{\infty}(G) \bar{\otimes} L^{\infty}(G)\right) \widehat{\otimes}\left(L^{\infty}(G) \bar{\otimes} L^{2}(G, H)_{c}\right)$ are complete contractions.

Corollary 4.7. Let $G$ be a locally compact group and $H$ be a Hilbert space. For $T \in \mathcal{B}\left(L^{2}(G, H)\right)$,

$$
\left\|\Phi_{2} \circ(1 \otimes T) \circ \Phi_{1}: M_{c b} A(G) \widehat{\otimes} L^{2}(G, H)_{c} \rightarrow L^{\infty}(G) \bar{\otimes} L^{2}(G, H)_{c}\right\|_{c b} \leq\|T\| .
$$

Proof of Theorem 4.1. Suppose $G$ has the AP. Note that for any $h \in A(G) \cap C_{c}(G)$,

$$
\left.\omega_{f_{i}, \bar{h}}\right|_{V N(G)}=h * \check{f}_{i}=h * f_{i} \in A(G),
$$

and $h * f_{i}(s)=\left\langle\lambda(s) \lambda\left(f_{i}\right) \eta, \zeta\right\rangle, s \in G$, where $\langle\lambda(\cdot) \eta, \zeta\rangle$ is a representation of $h$. Hence

$$
\left\|h * f_{i}-h\right\|_{A(G)} \rightarrow 0 .
$$

By the AP pick a net $\left(h_{j}\right)$ of real-valued functions in $A(G) \cap C_{c}(G)$ converging to 1 in the weak* topology of $M_{c b} A(G)$.

Fix $T \in G \bar{\rtimes} M$ and a positive $\rho \in \mathcal{T}\left(L^{2}(G) \otimes H\right)$. Write $\rho=\sum_{n=1}^{\infty} \xi_{n} \xi_{n}^{*}$ for a sequence $\left(\xi_{n}\right)$ of vectors in $L^{2}(G) \otimes H$ satisfying $\sum_{n=1}^{\infty}\left\|\xi_{n}\right\|^{2}=\|\rho\|$.

For every $n \in \mathbb{N}$ we have

$$
\left\|W_{12}\left(f_{i} \otimes \xi_{n}\right)-f_{i} \otimes \xi_{n}\right\|_{L^{1}(G) \otimes \gamma} \overline{L^{2}(G) \otimes H}=\int_{G} f_{i}(s)\left\|(\lambda(s) \otimes 1) \xi_{n}-\xi_{n}\right\| d s,
$$

where $\otimes^{\gamma}$ is the Banach space projective tensor product, so by Jensen's inequality

$$
\left\|W_{12}\left(f_{i} \otimes \xi_{n}\right)-f_{i} \otimes \xi_{n}\right\|_{L^{1}(G) \otimes \gamma}^{2} \overline{L^{2}(G) \otimes H} \leq \int_{G} f_{i}(s)\left\|(\lambda(s) \otimes 1) \xi_{n}-\xi_{n}\right\|^{2} d s .
$$

Given $\varepsilon>0$, pick $N \in \mathbb{N}$ such that $\sum_{n=N+1}^{\infty}\left\|\xi_{n}\right\|^{2}<\varepsilon / 8$. The function

$$
g_{\varepsilon}(s)=\sum_{n=1}^{N}\left\|(\lambda(s) \otimes 1) \xi_{n}-\xi_{n}\right\|^{2}
$$

is right uniformly continuous and satisfies $g_{\varepsilon}(e)=0$. Hence,

$$
\int_{G} f_{i}(s) g_{\varepsilon}(s)=g_{\varepsilon} * \check{f}_{i}(e)=g_{\varepsilon} * f_{i}(e) \rightarrow g_{\varepsilon}(e)=0 .
$$


Pick $i_{\varepsilon}$ such that

$$
\int_{G} f_{i}(s) g_{\varepsilon}(s)<\frac{\varepsilon}{2}
$$

for all $i \geq i_{\varepsilon}$. Then for all $i \geq i_{\varepsilon}$ we have

$$
\begin{aligned}
& \sum_{n=1}^{\infty}\left\|W_{12}\left(f_{i} \otimes \xi_{n}\right)-f_{i} \otimes \xi_{n}\right\|_{L^{1}(G) \otimes \gamma}^{2} \overline{L^{2}(G) \otimes H} \\
& \leq \sum_{n=1}^{\infty} \int_{G} f_{i}(s)\left\|(\lambda(s) \otimes 1) \xi_{n}-\xi_{n}\right\|^{2} d s \\
& =\sum_{n=1}^{N} \int_{G} f_{i}(s)\left\|(\lambda(s) \otimes 1) \xi_{n}-\xi_{n}\right\|^{2} d s+\sum_{n=N+1}^{\infty} \int_{G} f_{i}(s)\left\|(\lambda(s) \otimes 1) \xi_{n}-\xi_{n}\right\|^{2} d s \\
& \leq \int_{G} f_{i}(s)\left(\sum_{n=1}^{N}\left\|(\lambda(s) \otimes 1) \xi_{n}-\xi_{n}\right\|^{2}\right) d s+\sum_{n=N+1}^{\infty} 4\left\|\xi_{n}\right\|^{2} \\
& <\int_{G} f_{i}(s) g_{\varepsilon}(s) d s+\frac{\varepsilon}{2} \\
& <\varepsilon .
\end{aligned}
$$

Hence,

$$
\sum_{n=1}^{\infty}\left\|W_{12}\left(f_{i} \otimes \xi_{n}\right)-f_{i} \otimes \xi_{n}\right\|_{L^{1}(G) \otimes \gamma}^{2} \overline{L^{2}(G) \otimes H} \rightarrow 0
$$

Recall that on $C_{c}(G) \otimes L^{2}(G, H)$, the maps $\Phi_{1}$ and $\Phi_{2}$ coincide with $(\widehat{W} \otimes 1)$ and $\left(\widehat{W}^{*} \otimes 1\right)$, respectively. Corollary 4.7 then entails

$$
\begin{aligned}
& \left|\left\langle\widehat{\alpha}\left(u\left(f_{i}\right) T\right)\left(W_{12}\left(f_{i} \otimes \xi_{n}\right)-\left(f_{i} \otimes \xi_{n}\right)\right), h_{j} \otimes \xi_{n}\right\rangle\right| \\
& =\left|\left\langle W_{12}\left(f_{i} \otimes \xi_{n}\right)-\left(f_{i} \otimes \xi_{n}\right), \widehat{W}_{12}^{*}\left(1 \otimes T^{*} u\left(f_{i}\right)^{*}\right)\right) \widehat{W}_{12}\left(h_{j} \otimes \xi_{n}\right)\right\rangle \mid \\
& \leq\left\|W_{12}\left(f_{i} \otimes \xi_{n}\right)-\left(f_{i} \otimes \xi_{n}\right)\right\|_{\left.L^{1}(G) \widehat{\otimes} \overline{L^{2}(G, H)_{r}} \| \Phi_{2}\left(1 \otimes T^{*} u\left(f_{i}\right)^{*}\right)\right) \Phi_{1}\left(h_{j} \otimes \xi_{n}\right) \|_{L^{\infty}(G) \bar{\otimes} L^{2}(G, H)_{c}}}\left\|u\left(f_{i}\right)\right\|\|T\|\left\|h_{j} \otimes \xi_{n}\right\|_{M_{c b} A(G) \widehat{\otimes} L^{2}(G, H)_{c}} \\
& \leq\left\|W_{12}\left(f_{i} \otimes \xi_{n}\right)-\left(f_{i} \otimes \xi_{n}\right)\right\|_{L^{1}(G) \otimes \gamma} \overline{L^{2}(G, H)_{r}}\left\|W_{12}\left(f_{i} \otimes \xi_{n}\right)-\left(f_{i} \otimes \xi_{n}\right)\right\|_{L^{1}(G) \otimes \gamma} \overline{L^{2}(G, H)_{r}}\|T\|\left\|h_{j}\right\|_{M_{c b} A(G)}\left\|\xi_{n}\right\|_{L^{2}(G) \otimes H},
\end{aligned}
$$

where $L^{2}(G, H)_{r}$ refers to the row operator space structure of $L^{2}(G, H)$. Hence, for every $j$, we have

$$
\begin{aligned}
& \sum_{n=1}^{\infty}\left|\left\langle\widehat{\alpha}\left(u\left(f_{i}\right) T\right)\left(W_{12}\left(f_{i} \otimes \xi_{n}\right)-\left(f_{i} \otimes \xi_{n}\right)\right), h_{j} \otimes \xi_{n}\right\rangle\right| \\
& \leq\|T\|\left\|h_{j}\right\|_{M_{c b} A(G)} \sum_{n=1}^{\infty}\left\|W_{12}\left(f_{i} \otimes \xi_{n}\right)-\left(f_{i} \otimes \xi_{n}\right)\right\|_{L^{1}(G) \otimes \gamma} \overline{L^{2}(G, H)_{r}}\left\|\xi_{n}\right\| \\
& \leq\|T\|\left\|h_{j}\right\|_{M_{c b} A(G)}\left(\sum_{n=1}^{\infty}\left\|W_{12}\left(f_{i} \otimes \xi_{n}\right)-\left(f_{i} \otimes \xi_{n}\right)\right\|_{L^{1}(G) \otimes \gamma}^{2} \overline{L^{2}(G, H)_{r}}\right)^{1 / 2}\left(\sum_{n=1}^{\infty}\left\|\xi_{n}\right\|^{2}\right)^{1 / 2} \\
& \rightarrow 0 .
\end{aligned}
$$


Putting things together, for each $j$

$$
\begin{aligned}
& \lim _{i} \int_{G} \frac{h_{j}(x)}{\Delta(x)}\left\langle E\left(u\left(f_{i}\right) T u\left(f_{i}\right) u\left(x^{-1}\right)\right) u(x), \rho\right\rangle d x=\lim _{i}\left\langle\left(\omega_{f_{i}, h_{j}} \otimes \mathrm{id}\right)\left(\widehat{\alpha}\left(u\left(f_{i}\right) T\right) W_{12}\right), \rho\right\rangle \\
& =\lim _{i} \sum_{n=1}^{\infty}\left\langle\widehat{\alpha}\left(u\left(f_{i}\right) T\right) W_{12}\left(f_{i} \otimes \xi_{n}\right), h_{j} \otimes \xi_{n}\right\rangle=\lim _{i} \sum_{n=1}^{\infty}\left\langle\widehat{\alpha}\left(u\left(f_{i}\right) T\right)\left(f_{i} \otimes \xi_{n}\right), h_{j} \otimes \xi_{n}\right\rangle \\
& =\lim _{i} \sum_{n=1}^{\infty}\left\langle\left(\omega_{f_{i}, h_{j}} \otimes \mathrm{id}\right) \widehat{\alpha}\left(u\left(f_{i}\right) T\right) \xi_{n}, \xi_{n}\right\rangle=\lim _{i}\left\langle\left(h_{j} * f_{i} \otimes \mathrm{id}\right) \widehat{\alpha}\left(u\left(f_{i}\right) T\right), \rho\right\rangle=\left\langle\left(h_{j} \otimes \mathrm{id}\right) \widehat{\alpha}(T), \rho\right\rangle,
\end{aligned}
$$

where in the last equality we used the norm continuity of the map

$$
A(G) \ni \psi \mapsto(T \mapsto T \cdot \psi) \in \mathcal{C B}(G \bar{\rtimes} M) .
$$

Now, $\left(\left(h_{j} \otimes \mathrm{id}\right) \widehat{\alpha}(T)=T \cdot h_{j}=\left(\widehat{\Theta}\left(h_{j}\right) \otimes \mathrm{id}\right)(T)\right.$. Letting $m_{h_{j}}=\left.\widehat{\Theta}\left(h_{j}\right)\right|_{V N(G)} \in \mathcal{C B}^{\sigma}(V N(G))$ denote the canonical multiplication map on $V N(G)$, it follows from [21, Proposition 1.7, Theorem 1.9(b)] that $\left(m_{h_{j}} \otimes \operatorname{id}_{N}\right) \rightarrow \operatorname{id}_{V N(G) \bar{\otimes} N}$ point weak* for any von Neumann algebra $N$. By the proof of $[22$, Proposition 4.3] we have

$$
\left(\widehat{\Theta}\left(h_{j}\right) \otimes \mathrm{id}\right)(S)=\left(\omega_{0} \otimes \operatorname{id}_{\mathcal{B}\left(L^{2}(G)\right) \bar{\otimes} M}\right)\left(\widehat{W}_{12}\left(\left(m_{h_{j}} \otimes \operatorname{id}_{\mathcal{B}\left(L^{2}(G)\right) \bar{\otimes} M}\right)\left(\widehat{W}_{12}^{*} S_{23} \widehat{W}_{12}\right)\right) \widehat{W}_{12}^{*}\right)
$$

for all $S \in \mathcal{B}\left(L^{2}(G)\right) \bar{\otimes} M$, where $\omega_{0} \in \mathcal{T}\left(L^{2}(G)\right)$ is an arbitrary state. Hence,

$$
\lim _{j}\left\langle\left(h_{j} \otimes \mathrm{id}\right) \widehat{\alpha}(T), \rho\right\rangle=\lim _{j}\left\langle\left(\widehat{\Theta}\left(h_{j}\right) \otimes \mathrm{id}\right)(T), \rho\right\rangle=\langle T, \rho\rangle
$$

By polarization, the above analysis is valid for any trace class operator. Hence,

$$
T=w^{*}-\lim _{j} \lim _{i} \int \frac{h_{j}(x)}{\Delta(x)} E\left(u\left(f_{i}\right) T u\left(f_{i}\right) u\left(x^{-1}\right)\right) u(x) d x .
$$

Conversely, suppose (2) holds for every $W^{*}$-dynamical system $(M, G, \alpha)$. If $G$ acts trivially on a von Neumann algebra $M$, then for every $T \in G \bar{\rtimes} M=V N(G) \bar{\otimes} M$ we have

$$
T=w^{*}-\lim _{j} \lim _{i} \int_{G} \frac{h_{j}(x)}{\Delta(x)} E\left(u\left(f_{i}\right) T u\left(f_{i}\right) u\left(x^{-1}\right)\right) u(x) d x .
$$

As in the proof of Theorem 4.1, for each $j$ it follows that

$$
\left(\widehat{\Theta}\left(h_{j}\right) \otimes \mathrm{id}\right)(T)=w^{*}-\lim _{i} \int_{G} \frac{h_{j}(x)}{\Delta(x)} E\left(u\left(f_{i}\right) T u\left(f_{i}\right) u\left(x^{-1}\right)\right) u(x) d x .
$$

Thus, $T=w^{*}-\lim _{j}\left(\widehat{\Theta}\left(h_{j}\right) \otimes \mathrm{id}\right)(T)$, implying that $\left(\widehat{\Theta}\left(h_{j}\right)\right)$ converges to $\operatorname{id}_{V N(G)}$ in the stable point weak* topology. By [21, Theorem 1.9 (b)], $G$ has the AP.

As an immediate application, we obtain:

Corollary 4.8. Let $G$ be a locally compact group with the AP. Then every action of $G$ on a von Neumann algebra has the slice map property.

For inner amenable groups $G$ (e.g., $G$ discrete) the converse of Corollary 4.8 holds by Example 3.8 and [9, Corollary 4.8]. For discrete groups a similar result was also shown in [38]. After the first version of this paper appeared, the converse of Corollary 4.8 was established by Andreou [2, Theorem 5.12]. Hence, a locally compact group $G$ as the AP if and only if every $W^{*}$-dynamical system has the slice map property.

Analogous results hold in the setting of $C^{*}$-dynamical systems, where we obtain norm convergence of the Fejér representation. The proof relies on the following Lemma, which is inspired by [41, Lemma 2.4]. In preparation, for every $T \in G \rtimes A$ and $\varphi \in(G \rtimes A)^{*}$, let $\omega_{T, \varphi}: M_{c b} A(G) \rightarrow \mathbb{C}$ be the functional

$$
\omega_{T, \varphi}(v)=\langle(\widehat{\Theta}(v) \otimes \mathrm{id})(T), \varphi\rangle, \quad v \in M_{c b} A(G) .
$$


Lemma 4.9. For every $T \in G \rtimes A$, and $\varphi \in(G \rtimes A)^{*}$, the functional $\omega_{T, \varphi} \in Q_{c b}(G)$.

Proof. First suppose that $T=\tilde{\pi}_{u} \times u(f)$ for some $f \in C_{c}(G, A)$, and $\varphi \in(G \rtimes A)^{*}$ is positive. By [15, Lemme 3.2] there exists $v_{f} \in A(G) \cap C_{c}(G)$ in the span of compactly supported positive definite functions satisfying $v_{f} \equiv 1$ on $\operatorname{supp}(f)$. It follows that $T=\left(\widehat{\Theta}\left(v_{f}\right) \otimes \mathrm{id}\right)(T)$. Also, by [34, Corollary 7.6.9, Lemma 7.7.6] we have $\left(\widehat{\Theta}\left(v_{f}\right) \otimes \mathrm{id}\right)_{*}(\varphi) \in(G \rtimes A)_{*}^{\prime \prime}$.

If $v_{i} \rightarrow v$ in $\sigma\left(M_{c b} A(G), Q_{c b}(G)\right)$ then, as above, $\left(\widehat{\Theta}\left(v_{i}\right) \otimes\right.$ id $)(S) \rightarrow(\widehat{\Theta}(v) \otimes$ id $)(S)$ weak* for all $S \in \mathcal{B}\left(L^{2}(G)\right) \bar{\otimes} \mathcal{B}\left(H_{u}\right)$, so that

$$
\begin{aligned}
\omega_{T, \varphi}\left(v_{i}\right) & =\left\langle\left(\widehat{\Theta}\left(v_{i}\right) \otimes \mathrm{id}\right)(T), \varphi\right\rangle=\left\langle\left(\widehat{\Theta}\left(v_{i}\right) \otimes \mathrm{id}\right)(T),\left(\widehat{\Theta}\left(v_{f}\right) \otimes \mathrm{id}\right)_{*}(\varphi)\right\rangle \\
& \rightarrow\left\langle(\widehat{\Theta}(v) \otimes \mathrm{id})(T),\left(\widehat{\Theta}\left(v_{f}\right) \otimes \mathrm{id}\right)_{*}(\varphi)\right\rangle=\langle(\widehat{\Theta}(v) \otimes \mathrm{id})(T), \varphi\rangle .
\end{aligned}
$$

Hence, $\omega_{T, \varphi} \in Q_{c b}(G)$. By norm density of $\tilde{\pi}_{u} \times u\left(C_{c}(G, A)\right)$ in $G \rtimes A$ and polarization, we see that $\omega_{T, \varphi} \in Q_{c b}(G)$ for every $T \in G \rtimes A$, and $\varphi \in(G \rtimes A)^{*}$.

Theorem 4.10. Let $G$ be a locally compact group, and denote by $\left(f_{i}\right) \subseteq C_{c}(G)_{\|\cdot\|_{1}=1}^{+}$a symmetric bounded approximate identity for $L^{1}(G)$. Then the following are equivalent.

(1) G has the AP.

(2) There exists a net $\left(h_{j}\right)$ in $A(G) \cap C_{c}(G)$ such that for every $C^{*}$-dynamical system $(A, G, \alpha)$,

$$
T=\lim _{j} \lim _{i} \int_{G} \frac{h_{j}(x)}{\Delta(x)} E\left(u\left(f_{i}\right) T u\left(f_{i}\right) u\left(x^{-1}\right)\right) u(x) d x, \quad T \in G \rtimes A .
$$

Proof. The proof goes through more or less unchanged from that of Theorem 4.1. The final convergence simply needs to be upgraded to the norm topology. If $G$ has the AP, let $\left(h_{j}\right)$ be a net of real-valued functions in $A(G) \cap C_{c}(G)$ converging to 1 in the weak* topology of $M_{c b} A(G)$. Then as above, for each $j$ we have

$$
\lim _{i} \int_{G} \frac{h_{j}(x)}{\Delta(x)}\left\langle E\left(u\left(f_{i}\right) T u\left(f_{i}\right) u\left(x^{-1}\right)\right) u(x), \rho\right\rangle d x=\left\langle\left(h_{j} \otimes \mathrm{id}\right) \widehat{\alpha}(T), \rho\right\rangle=\left\langle\left(\widehat{\Theta}\left(h_{j}\right) \otimes \mathrm{id}\right)(T), \rho\right\rangle,
$$

for all $T \in G \rtimes A$ and $\rho \in \mathcal{T}\left(L^{2}(G) \otimes H_{u}\right)$, where the convergence is uniform in $\rho$. Hence,

$$
\lim _{i} \int_{G} \frac{h_{j}(x)}{\Delta(x)} E\left(u\left(f_{i}\right) T u\left(f_{i}\right) u\left(x^{-1}\right)\right) u(x) d x=\left(\widehat{\Theta}\left(h_{j}\right) \otimes \mathrm{id}\right)(T)
$$

in the norm topology of $\mathcal{B}\left(L^{2}(G) \otimes H_{u}\right)$. By Lemma 4.9

$$
\left\langle\left(\widehat{\Theta}\left(h_{j}\right) \otimes \mathrm{id}\right)(T), \varphi\right\rangle=\omega_{T, \varphi}\left(h_{j}\right) \rightarrow \omega_{T, \varphi}(1)=\langle T, \varphi\rangle
$$

for any $\varphi \in(G \rtimes A)^{*}$, that is, $\left(\widehat{\Theta}\left(h_{j}\right) \otimes \mathrm{id}\right)(T) \rightarrow T$ weakly in $G \rtimes A$. Taking convex combinations of the $h_{j}$, we may assume that the later convergence is relative to the norm topology. Hence,

$$
\lim _{j} \lim _{i} \int_{G} \frac{h_{j}(x)}{\Delta(x)} E\left(u\left(f_{i}\right) T u\left(f_{i}\right) u\left(x^{-1}\right)\right) u(x) d x=T
$$

in the norm topology of $G \rtimes A$.

Conversely, suppose (2) holds for every $C^{*}$-dynamical system $(A, G, \alpha)$, and let $G$ act trivially on $\mathcal{K}(H)$ for a separable Hilbert space $H$. Then for every $T \in G \rtimes A=C_{\lambda}^{*}(G) \otimes^{\vee} \mathcal{K}(H)$ we have

$$
T=\lim _{j} \lim _{i} \int_{G} \frac{h_{j}(x)}{\Delta(x)} E\left(u\left(f_{i}\right) T u\left(f_{i}\right) u\left(x^{-1}\right)\right) u(x) d x .
$$

As in the proof of Theorem 4.10, for each $j$ it follows that

$$
\left(\widehat{\Theta}\left(h_{j}\right) \otimes \mathrm{id}\right)(T)=\lim _{i} \int_{G} \frac{h_{j}(x)}{\Delta(x)} E\left(u\left(f_{i}\right) T u\left(f_{i}\right) u\left(x^{-1}\right)\right) u(x) d x .
$$


Thus, $T=\lim _{j}\left(\widehat{\Theta}\left(h_{j}\right) \otimes \mathrm{id}\right)(T)$, implying that $\left(\widehat{\Theta}\left(h_{j}\right)\right)$ converges to $\mathrm{id}_{C_{\lambda}^{*}(G)}$ in the stable point norm topology. By [21, Theorem 1.9 (c)], $G$ has the AP.

Corollary 4.11. Let $G$ be a locally compact group with the AP. Then any action of $G$ on any $C^{*-}$ algebra has the slice map property. In particular, $C_{\lambda}^{*}(G)$ has the strong operator space approximation property (SOAP).

At a similar time as the first version of this paper, it was shown by Suzuki that for any $C^{*}$ dynamical system $(A, G, \alpha)$ where $G$ has the AP, $G \rtimes A$ has the SOAP if and only if $A$ has the SOAP [39, Theorem 3.6]. In particular, this yields a different proof that $C_{\lambda}^{*}(G)$ has the SOAP for any locally compact group $G$ with the AP. Contrary to the $W^{*}$-setting (see Corollary 4.8 and the subsequent discussion), the converse of Corollary 4.11 remains open in general.

\section{Applications}

5.1. The approximation property and exactness. It is well-known that a discrete group $G$ with the AP is exact [21]. The argument proceeds by establishing the SOAP of $C_{\lambda}^{*}(G)$, then appealing to the fact that $C^{*}$-algebras with the SOAP are exact, and the fact that a discrete group $G$ with $C_{\lambda}^{*}(G)$ exact is necessarily exact [23, Theorem 5.2]. To the authors' knowledge, a similar argument through the operator space structure of $C_{\lambda}^{*}(G)$ cannot be applied in the general locally compact setting. However, combining Corollary 4.11 with Proposition 3.14 we see that the implication persists. This answers Problem 9.4 (1) in [27].

Theorem 5.1. A locally compact group with the AP is exact.

Remark 5.2. The result of Theorem 5.1 also appears in the independent very recent work of Suzuki [39], of which we became aware after obtaining this result.

Remark 5.3. It was shown in [7, Corollary E] that a weakly amenable second countable locally compact group is exact. We therefore obtain a different proof of this fact, valid for any locally compact group.

5.2. The Fejér property for discrete dynamical systems. Let $(A, G, \alpha)$ be a $C^{*}$-dynamical system with $G$ discrete. Following [4], a function $F: G \times A \rightarrow A$, linear in the second variable, is a (completely) bounded multiplier of $(A, G, \alpha)$ if

$$
M_{F}: G \rtimes A \ni \sum_{x \in G} a_{x} u(x) \mapsto \sum_{x \in G} F\left(x, a_{x}\right) u(x) \in G \rtimes A
$$

extends to a (completely) bounded map. A function $F: G \times A \rightarrow A$ has finite $G$-support if $F(x, \cdot)=0$ for all but finitely many $x \in G$. The $C^{*}$-dynamical system $(A, G, \alpha)$ has the Fejér property if there exists a net $\left(F_{i}\right)$ of bounded multipliers for which each $F_{i}$ has finite $G$-support and $M_{F_{i}}(T) \rightarrow T$ in norm for all $T \in G \rtimes A$. In [4, Theorem 5.6] the authors show that if $G$ is weakly amenable, then any $C^{*}$-dynamical system has the Fejér property, and they ask whether the corresponding result is true for groups with the AP.

Theorem 5.4. Let $G$ be a discrete group with the AP. Then every $C^{*}$-dynamical system $(A, G, \alpha)$ has the Fejér property.

Proof. By Theorem 4.10 there exists a net $\left(h_{i}\right) \subseteq A(G) \cap C_{c}(G)$ such that

$$
T=\lim _{j} \sum_{x \in G} h_{j}(x) E\left(T u\left(x^{-1}\right)\right) u(x), \quad T \in G \rtimes A .
$$

Letting $F_{j}(x, a)=h_{j}(x) a, x \in G, a \in A$, we obtain a net $\left(F_{j}\right)$ of finitely $G$-supported functions $G \times A \rightarrow A$ whose corresponding multipliers $M_{F_{j}}$ equal $\left(\Theta\left(h_{j}\right) \otimes \mathrm{id}\right)$ (this is easily verified on finitely 
supported elements of the form $\sum_{x \in G} a_{x} u(x)$, the extension to $G \rtimes A$ following from boundedness). The proof of Theorem 4.10 then shows

$$
M_{F_{i}}(T)=\left(\Theta\left(h_{j}\right) \otimes \mathrm{id}\right)(T)=\sum_{x \in G} h_{j}(x) E\left(T u\left(x^{-1}\right)\right) u(x), \quad T \in G \rtimes A .
$$

Hence, $M_{F_{i}}(T) \rightarrow T$ for all $T \in G \rtimes A$ and $(A, G, \alpha)$ has the Fejér property.

Alternatively, Theorem 5.4 can be deduced from the proof of [38, Proposition 3.4], as pointed out to us by Suzuki, who learned about this question and our solution through an earlier version of the present paper.

It is natural to consider a generalization of the Fejér property for locally compact groups using the recent theory of Herz-Schur multipliers of dynamical systems [29], and connect this to our slice map property for actions. This and related questions will appear in subsequent work.

5.3. Structure of $V N(G)$-bimodules. Given a locally compact group $G$ and a closed left ideal $J \triangleleft L^{1}(G)$, following [3], we let

$$
\operatorname{Ran}(J)=\overline{\operatorname{span}\left\{\Theta^{r}(f)_{*}(\rho) \mid f \in J, \rho \in \mathcal{T}\left(L^{2}(G)\right)\right\}}{ }^{\|\cdot\|_{\mathcal{T}\left(L^{2}(G)\right)}}
$$

and

$$
\operatorname{Bim}\left(J^{\perp}\right)=\overline{\operatorname{span}\left\{x M_{f} y \mid f \in J^{\perp}, x, y \in V N(G)\right\}}{ }^{w^{*}},
$$

where $\Theta^{r}(f)_{*}$ is the pre-adjoint of the normal completely bounded map

$$
\Theta^{r}(f): \mathcal{B}\left(L^{2}(G)\right) \ni T \mapsto \int_{G} f(s) \rho(s) T \rho\left(s^{-1}\right) d s \in \mathcal{B}\left(L^{2}(G)\right) .
$$

In [3] it was shown that for any $G$ and $J \triangleleft L^{1}(G), \operatorname{Bim}\left(J^{\perp}\right) \subseteq \operatorname{Ran}(J)^{\perp}$. The reverse inclusion was established in a few special cases, including abelian, compact, and weakly amenable discrete groups. The authors asked whether it holds in general. As an application of Theorem 4.1, we now show that the reverse inclusion holds for all locally compact groups with the AP.

Theorem 5.5. Let $G$ be a locally compact group with the AP. Then for any closed left ideal $J \triangleleft L^{1}(G)$ we have $\operatorname{Bim}\left(J^{\perp}\right)=\operatorname{Ran}(J)^{\perp}$.

Proof. First, we make the identification $\mathcal{B}\left(L^{2}(G)\right) \cong G \bar{\rtimes} L^{\infty}(G)$ via the extended (right) co-product

$$
\Gamma^{r}: \mathcal{B}\left(L^{2}(G)\right) \ni T \mapsto V(T \otimes 1) V^{*} \in \mathcal{B}\left(L^{2}(G)\right) \bar{\otimes} L^{\infty}(G) .
$$

Under this identification it follows that the dual co-action $\widehat{\alpha}: \mathcal{B}\left(L^{2}(G)\right) \rightarrow V N(G) \bar{\otimes} \mathcal{B}\left(L^{2}(G)\right)$ is precisely the extended (left) co-product

$$
\widehat{\Gamma}^{l}: \mathcal{B}\left(L^{2}(G)\right) \ni T \mapsto \widehat{W}^{*}(1 \otimes T) \widehat{W} \in V N(G) \bar{\otimes} \mathcal{B}\left(L^{2}(G)\right) .
$$

Next, for any $r, s, t \in G$, and $\xi \in L^{2}(G \times G)$ we have

$$
\begin{aligned}
(\lambda(r) \otimes \rho(r)) \widehat{W} \xi(s, t) & =\widehat{W} \xi\left(r^{-1} s, t r\right) \Delta(r)^{1 / 2} \\
& =\xi(t s, t r) \Delta(r)^{1 / 2} \\
& =(1 \otimes \rho(r)) \xi(t s, t) \\
& =\widehat{W}(1 \otimes \rho(r)) \xi(s, t) .
\end{aligned}
$$

Thus, $(\lambda(r) \otimes \rho(r)) \widehat{W}=\widehat{W}(1 \otimes \rho(r))$. For any $r \in G$, positive definite $\psi \in A(G)$, and $T \in \mathcal{B}\left(L^{2}(G)\right)$ we therefore have

$$
\begin{aligned}
\operatorname{Ad}(\rho(r))((\psi \otimes \mathrm{id}) \widehat{\alpha}(T)) & =(\psi \otimes \mathrm{id})\left((1 \otimes \rho(r)) \widehat{W}^{*}(1 \otimes T) \widehat{W}\left(1 \otimes \rho\left(r^{-1}\right)\right)\right) \\
& =(\psi \otimes \mathrm{id})\left(\widehat{W}^{*}\left(1 \otimes \rho(r) T \rho\left(r^{-1}\right)\right) \widehat{W}\right) \\
& =(\psi \otimes \mathrm{id}) \widehat{\alpha}\left(\rho(r) T \rho\left(r^{-1}\right)\right)
\end{aligned}
$$


In the notation of [19, Theorem 3.1 (a)], the above reads $E_{\psi} \circ \operatorname{Ad}(\rho(r))=\operatorname{Ad}(\rho(r)) \circ E_{\psi}$. Since the operator-valued weight $E=(\varphi \otimes$ id $) \circ \widehat{\alpha}$ coincides with Haagerup's construction in [19], it satisfies

$$
E=\sup _{\psi<<\delta_{e}} E_{\psi}
$$

where $\psi$ ranges through the positive definite elements of $A(G)$ and $<<$ is the standard positive definite ordering (see [19, Theorem 3.1 (b), Proposition 2.4]). It follows that for every $f, g \in C_{c}(G)$ and $T \in \mathcal{B}\left(L^{2}(G)\right)$,

$$
\rho(r) E(\lambda(f) T \lambda(g)) \rho\left(r^{-1}\right)=E\left(\lambda(f) \rho(r) T \rho\left(r^{-1}\right) \lambda(g)\right), \quad r \in G,
$$

and hence, by normality of $E(\lambda(f)(\cdot) \lambda(g))$, that

$$
\Theta^{r}(h)(E(\lambda(f) T \lambda(g)))=\int_{G} h(r) \rho(r) E(\lambda(f) T \lambda(g)) \rho\left(r^{-1}\right)=E\left(\lambda(f) \Theta^{r}(h)(T) \lambda(g)\right)
$$

for all $h \in L^{1}(G)$.

Now, suppose $T \in \operatorname{Ran}(J)^{\perp}$. As $\operatorname{Ran}(J)^{\perp}$ is a $V N(G)$-bimodule, we have $\lambda(f) T \lambda(f) \lambda\left(x^{-1}\right) \in$ $\operatorname{Ran}(J)^{\perp}$ for all $f \in C_{c}(G)$ and $x \in G$. Since $\operatorname{Ran}(J)^{\perp}=\operatorname{Ker}\left(\Theta^{r}(J)\right)$ [3, Lemma 3.1], and

$$
\Theta^{r}(h)\left(E\left(\lambda(f) T \lambda(f) \lambda\left(x^{-1}\right)\right)\right)=E\left(\lambda(f) \Theta^{r}(h)(T) \lambda(f) \lambda\left(x^{-1}\right)\right)=0
$$

for all $h \in J$, we have $E\left(\lambda(f) T \lambda(f) \lambda\left(x^{-1}\right)\right) \in \operatorname{Ran}(J)^{\perp} \cap L^{\infty}(G)=J^{\perp}$ by [3, Lemma 3.3]. The Fejér representation for $T$ from Theorem 4.1 then implies that $T \in \operatorname{Bim}\left(J^{\perp}\right)$.

After the first version of this paper appeared, Theorem 5.5 was obtained using different techniques by Andreou [1, Remark 5.4].

\section{ACKNOWLEDGEMENTS}

The authors are grateful for the reviewers' comments, which improved the overall presentation of the paper. The authors would also like to thank Mahmood Alaghmandan, Mehrdad Kalantar, Hung-Chang Liao, and Ivan Todorov for helpful discussions at various points during the project. The first author was partially supported by the NSERC Discovery Grant RGPIN-2017-06275, and the second author was partially supported by the NSERC Discovery Grant RGPIN-2014-06356.

\section{REFERENCES}

[1] D. Andreou Crossed products of dual operator spaces by locally compact groups. arXiv:1910.00433.

[2] D. Andreou Crossed products of dual operator spaces and a characterization of groups with the approximation property. arXiv:2004.07169.

[3] M. Anoussis, A. Katavolos and I. G. Todorov, Bimodules over $V N(G)$, harmonic operators and the noncommutative Poisson boundary. Studia Math. 249 (2019), no. 2, 193-213.

[4] E. Bédos and R. Conti, Fourier series and twisted $C^{*}$-crossed products. J. Fourier Anal. Appl. 21 (2015), no. 1, 32-75.

[5] E. Bédos and R. Conti, The Fourier-Stieltjes algebra of a $C^{*}$-dynamical system. Internat. J. Math. 27 (2016), no. $6,50 \mathrm{pp}$.

[6] D. P. Blecher and R. R. Smith, The dual of the Haagerup tensor product. J. London Math. Soc. (2) 45 (1992), no. 1, 126-144.

[7] J. Brodzki, C. Cave, and K. Li, Exactness of locally compact groups. Adv. Math. 312 (2017), 209-233.

[8] J. Crann, Amenability and covariant injectivity of locally compact quantum groups II. Canadian J. Math. 69 (2017), no. 5, 1064-1086.

[9] J. Crann, Inner amenability and approximation properties of locally compact quantum groups. Indiana Univ. Math. J. 68 (2019), no. 6, 1721-1766.

[10] J. de Cannière and U. Haagerup, Multipliers of the Fourier Algebras of Some Simple Lie Groups and Their Discrete Subgroups. American J. Math., 107 (1985), no. 2, 455-500.

[11] Z. Dong and Z.-J. Ruan, A Hilbert module approach to the Haagerup property. Integral Equations Operator Theory 73 (2012), no. 3, 431-454. 
[12] E. G. Effros and Z.-J. Ruan, Operator spaces. London Mathematical Society Monographs. New Series, 23. The Clarendon Press, Oxford University Press, New York, 2000.

[13] E. G. Effros and Z.-J. Ruan, Operator space tensor products and Hopf convolution algebras. J. Operator Theory 50 (2003), no. 1, 131-156.

[14] R. Exel, Amenability for Fell bundles. J. Reine Angew. Math. 492 (1997), 41-73.

[15] P. Eymard, L'algèbre de Fourier d'un groupe localement compact. Bull. Soc. Math. France 92 (1964) 181-236.

[16] L. Fejér, Untersuchungen über Fouriersche Reihen. Math. Ann. 58 (1903), no. 1-2, 51-69.

[17] L. Fejér, Sur les singularités de la série de Fourier des fonctions continues. Ann. Sci. École Norm. Sup. (3) 28 (1911), 63-104.

[18] A. Guichardet, Caractères des algèbres de Banach involutives. Ann. Inst. Fourier (Grenoble) 13 (1963), 1-81.

[19] U. Haagerup, On the dual weights for crossed products of von Neumann algebras. II. Application of operatorvalued weights. Math. Scand. 43 (1978/79), no. 1, 119-140.

[20] U. Haagerup, Operator-valued weights in von Neumann algebras I. J. Funct. Anal. 32 (1979), no. 2, 175-206.

[21] U. Haagerup and J. Kraus, Approximation properties for group $C^{*}$-algebras and group von Neumann algebras. Trans. Amer. Math. Soc. 44 (1994), no. 2, 667-699.

[22] M. Junge, M. Neufang and Z.-J. Ruan, A representation theorem for locally compact quantum groups. Internat. J. Math. 20 (2009), no. 3, 377-400.

[23] E. Kirchberg and S. Wassermann, Exact groups and continuous bundles of $C^{*}$-algebras. Math. Ann. 315 (1999), no. 2, 169-203.

[24] J. Kraus and Z-J. Ruan, Multipliers of Kac algebras. International J. Math. 8 (1996), 213-248.

[25] J. Kustermans and S. Vaes, Locally compact quantum groups in the von Neumann algebraic setting. Math. Scand. $92(2003)$, no. $1,68-92$.

[26] M. B. Landstad, Duality theory for covariant systems. Trans. Amer. Math. Soc. 248 (1979), no. 2, $223-267$.

[27] K. Li, Property A and coarse embedding for locally compact groups. PhD thesis, University of Copenhagen (2015).

[28] A. McKee, Weak amenability for dynamical systems. arXiv:1612.01758.

[29] A. McKee, I.G. Todorov and L. Turowska, Herz-Schur multipliers of dynamical systems. Adv. Math. 331 (2018), 387-438.

[30] A. McKee, A. Skalski, I.G. Todorov and L. Turowska, Positive Herz-Schur multipliers and approximation properties of crossed products. Math. Proc. Cambridge Philos. Soc. 165 (2018), no. 3, 511-532.

[31] R. Mercer, Convergence of Fourier series in discrete crossed products of von Neumann algebras. Proc. Amer. Math. Soc. 94 (1985), no. 2, 254-258.

[32] M. Neufang, Z.-J. Ruan and N. Spronk, Completely isometric representations of $M_{c b} A(G)$ and $U C B(\widehat{G})^{*}$. Trans. Amer. Math. Soc. 360 (2008), no. 3, 1133-1161.

[33] A. L. T., Paterson, Amenability. Mathematical Surveys and Monographs 29, American Mathematical Society, Providence, Rhode Island, 1988.

[34] G. K. Pedersen, $C^{*}$-algebras and their automorphism groups. London Mathematical Society Monographs, 14. Academic Press, Inc. London-New York, 1979.

[35] A. Sierakowski, The ideal structure of reduced crossed products. Münster J. Math. 3 (2010), 237-262.

[36] N. Spronk, Measurable Schur multipliers and completely bounded multipliers of the Fourier algebras. Proc. London Math. Soc. 89 (2004), 161-192.

[37] S. Strătilă. Modular theory in operator algebras. Editura Academiei and Abacus Press, 1981.

[38] Y. Suzuki, Group $C^{*}$-algebras as decreasing intersection of nuclear $C^{*}$-algebras. Amer. J. Math. 139 (2017), no. 3, 681-705.

[39] Y. Suzuki, The approximation property and exactness of locally compact groups. J. Math. Soc. Japan. to appear. arXiv:1901.07430.

[40] O. Uuye and J. Zacharias, The Fubini product and its applications. arXiv: 1603.04527

[41] J. Zacharias, On the invariant translation approximation property for discrete groups. Proc. Amer. Math. Soc. 134 (2006), no. 7, 1909-1916.

[42] G. Zeller-Meier, Produits croisés d'une $C^{*}$-algèbre par un groupe d'automorphismes. J. Math. Pures Appl. 47 (1968), 101-239.

E-mail address: jasoncrann@cunet.carleton.ca

E-mail address: matthias.neufang@carleton.ca

${ }^{1}$ School of Mathematics and Statistics, Carleton University, Ottawa, ON, Canada H1S 5B6

${ }^{2}$ Université Lille 1 - Sciences et Technologies, UFR de Mathématiques, Laboratoire de Mathématiques Paul Painlevé - UMR CNRS 8524, 59655 Villeneuve D’AscQ Cédex, France 